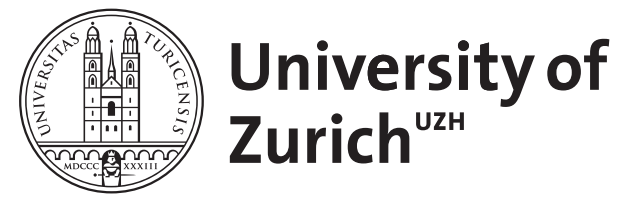

\title{
Cycle groups for Artin stacks
}

Kresch, A

\begin{abstract}
We construct an algebraic homology functor for Artin stacks of finite type over a field, and we develop intersection-theoretic properties.
\end{abstract}

DOI: https://doi.org/10.1007/s002220050351

Posted at the Zurich Open Repository and Archive, University of Zurich ZORA URL: https://doi.org/10.5167/uzh-22143

Journal Article

Accepted Version

Originally published at:

Kresch, A (1999). Cycle groups for Artin stacks. Inventiones Mathematicae, 138(3):495-536.

DOI: https://doi.org/10.1007/s002220050351 


\title{
Cycle groups for Artin stacks
}

\author{
Andrew Kresch ${ }^{1}$ \\ 28 October 1998
}

\section{Contents}

1 Introduction $\quad 2$

2 Definition and first properties 3

2.1 The homology functor . . . . . . . . . . . . . . . . . 3

2.2 Basic operations on Chow groups . . . . . . . . . . . . . . . . 8

2.3 Results on sheaves and vector bundles . . . . . . . . . . . . . . 10

2.4 The excision sequence . . . . . . . . . . . . . . . . . . . 11

3 Equivalence on bundles $\quad 12$

3.1 Trivial bundles . . . . . . . . . . . . . . . . . . . . . . . 12

3.2 The top Chern class operation . . . . . . . . . . . . . . . . . . 14

3.3 Collapsing cycles to the zero section . . . . . . . . . . . . . . . 15

3.4 Intersecting cycles with the zero section . . . . . . . . . . . . 17

3.5 Affine bundles . . . . . . . . . . . . . . . . . . . . . 19

3.6 Segre and Chern classes and the projective bundle theorem . . . . . . 20

4 Elementary intersection theory $\quad 22$

4.1 Fulton-MacPherson construction for local immersions . . . . . . . . . 22

4.2 Exterior product . . . . . . . . . . . . . . . . 24

4.3 Intersections on Deligne-Mumford stacks . . . . . . . . . . . . . . 24

4.4 Boundedness by dimension . . . . . . . . . . . . . . . . . . . . . . . . . . . . 25

4.5 Stratifications by quotient stacks . . . . . . . . . . . 26

5 Extended excision axiom $\quad 29$

5.1 A first higher Chow theory . . . . . . . . . . . . . . . . 29

5.2 The connecting homomorphism . . . . . . . . . . . . . 34

5.3 Homotopy invariance for vector bundle stacks . . . . . . . . . . 36

\footnotetext{
${ }^{1}$ Funded by a Fannie and John Hertz Foundation Fellowship for Graduate Study and an Alfred P. Sloan Foundation Dissertation Fellowship
} 
$6 \quad$ Intersection theory $\quad 37$

6.1 Intersections on Artin stacks . . . . . . . . . . . . . . . 37

6.2 Virtual fundamental class . . . . . . . . . . . . . . . . . . . . . . . . . 39

6.3 Localization formula . . . . . . . . . . . . . . . . . . . . . . . 39

\section{Introduction}

We define a Chow homology functor $A_{*}$ for Artin stacks and prove that it satisfies some of the basic properties expected from intersection theory. Consequences include an integer-valued intersection product on smooth Deligne-Mumford stacks, an affirmative answer to the conjecture that any smooth stack with finite but possibly nonreduced point stabilizers should possess an intersection product (this provides a positive answer to Conjecture 6.6 of [V2]), and more generally an intersection product (also integer-valued) on smooth Artin stacks which admit stratifications by global quotient stacks.

The definition presented here generalizes existing definitions. For representable stacks (i.e., algebraic spaces), the functor $A_{*}$ defined here reproduces the standard Chow groups. For Deligne-Mumford stacks [D-M] the functor differs by torsion from the naïve Chow groups (algebraic cycles modulo rational equivalence). The naïve Chow groups lead to a $\mathbb{Q}$-valued intersection theory on Deligne-Mumford stacks [Gi1, V2]. However, there is evidence that the naïve Chow group functor is not the correct object to work with if we wish to have integer coefficients. For instance, with the naïve Chow groups, there is no integer-valued intersection product on smooth DeligneMumford stacks. Even Chern classes of vector bundles do not exist except with rational coefficients.

Many Deligne-Mumford stacks are also global quotient stacks, and for a general global quotient stack, the functor $A_{*}$ reproduces the equivariant Chow groups of Dan Edidin and William Graham [E-G1]. In fact, the realization that the equivariant Chow groups are the correct groups to work with provided the starting point for the definition presented here. The main idea of [E-G1] is that a global quotient stack possesses a vector bundle $E$ such that the total spaces of $E^{\oplus n}$ (sums of $n$ copies of $E$ ) become increasingly well approximated by algebraic spaces as $n$ gets large. In any fixed codimension, the (naïve) Chow groups of these approximating spaces stabilize, and serve as the equivariant Chow homology functor.

This preprint takes the idea further by considering cycle classes in all vector bundles on an Artin stack. We say that a bundle $F$ dominates $E$ if there exists a vector bundle surjection $\varphi: F \rightarrow E$. Once we know that corresponding pullback map on cycle classes is independent of the particular choice of $\varphi$, we can take the direct limit of over all vector bundles. This gives us, for global quotient stacks, the equivariant Chow groups. In general, we obtain groups $\widehat{A}_{*} X$, but these groups themselves do not provide intersection theory. One can produce a stack $X$ which has no nontrivial vector bundles, but which has a closed substack $Y$ which is a global 
quotient. There is no way to push forward a nontrivial class $\alpha \in \widehat{A}_{*} Y$ via the inclusion map $i$ : $Y \rightarrow X$. The only thing to do is to define $i_{*} \alpha$ to be the formal pair $(i, \alpha)$. The set of all such pairs, modulo what is more or less the weakest possible equivalence relation which guarantees functoriality of pushforward, forms a group $A_{*} X$ (section 2.1) which provides a reasonable amount of intersection theory.

Once we have proved some basic properties for $A_{*}$ (sections 2.2-2.4 and section 3 ), we can obtain an integer-valued intersection product on smooth Deligne-Mumford stacks (section 4). All we need for this are standard constructions and properties from intersection theory. By way of constrast, intersection theory on Artin stacks requires homotopy invariance for objects more general than vector bundles. These objects, called vector bundle stacks in $[\mathrm{B}-\mathrm{F}]$, look locally like a quotient of of one vector bundle by the (additive) action of another. The proof of homotopy invariance for vector bundle stacks uses a localization argument (section 5), and once we have this property for a class of stacks then intersection theory follows. The relevant class of stacks consists of stacks which can be stratified by locally closed substacks that are global quotients. So, any such stack, if it is smooth, possesses an integervalued intersection product (section 6.1). This class of stacks includes (finite-type approximations of) many interesting stacks such as moduli stacks of stable or prestable curves and moduli stacks of vector bundles.

The paper $[\mathrm{B}-\mathrm{F}]$ constructs a virtual fundamental class of the expected dimension from a perfect obstruction theory. This class is gotten as the "intersection with the zero section" of a cone stack sitting in some vector bundle stack. Lacking intersection theory on Artin stacks (a vector bundle stack is an Artin stack), the authors were forced to impose a technical hypothesis in order to carry out their construction. The construction can now be done in general (section 6.2). We conclude this preprint with a discussion (section 6.3) of the localization formula for torus actions.

The author is greatly indebted to his thesis advisor William Fulton, who provided constant guidance and assistance during the course of this project. The author would like to acknowledge helpful discussions with Dan Edidin, Henri Gillet, and Charles Walter. Much of the work was done at the Mittag-Leffler Institute during the 199697 program in algebraic geometry. The author would like to thank the staff and organizers there for providing a fantastic research environment.

\section{Definition and first properties}

\subsection{The homology functor}

We start by recalling the notion of algebraic cycle and rational equivalence on a stack and by introducing the functor $A_{*}^{\circ}$ of cycles modulo rational equivalence. From this we build the functor $A_{*}$ by a succession of direct limits. The first limit is over vector bundles (Definition 2.1.3, below). The second limit is over projective morphisms to the target stack (Definition 2.1.2 (ii)). As is always the case when we wish to take 
a limit of abelian groups indexed by a directed set which comes from a category by collapsing morphisms, we must take care to verify that the induced map on groups is independent of the choice of morphism whenever more than one morphism exists between two objects of the category (Remark 2.1.5 for the limit over vector bundles and Remark 2.1.10 for the limit over projective morphisms).

Convention 2.1.1. All stacks are Artin stacks (i.e., algebraic stacks with smooth atlases $[\mathrm{A}, \mathrm{L}-\mathrm{MB}]$ ) and are of finite type over a fixed base field. All morphisms are morphisms over the base field. All regular (local) immersions are of constant codimension (a local immersion is by definition a representable unramified morphism; to every local immersion there is an associated normal cone, and if the cone is a bundle then the morphism is called a regular local immersion [V2]). A morphism of stacks $X \rightarrow Y$ is called projective if it can be factored (up to 2-isomorphism) as a closed immersion followed by the projection morphism $\mathbf{P}(\mathcal{E}) \rightarrow Y$ coming from a coherent sheaf $\mathcal{E}$ of $\mathcal{O}_{Y}$-modules on $Y$ [EGA2, L-MB]. Closed immersions and projections of the form $\mathbf{P}(\mathcal{E}) \rightarrow Y$ are examples of representable morphisms, so that every projective morphism of stacks is representable as well.

Let us recall that if $Y$ is a stack, then there is a category, known as the category of $Y$-stacks, whose objects consist of pairs $(X, f)$ with $X$ a stack (in our case, according to Convention 2.1.1, always of finite type over the base field) and $f$ a representable morphism from $X$ to $Y$. A morphism from $(X, f)$ to $\left(X^{\prime}, f^{\prime}\right)$ is a pair $(\varphi, \alpha)$, where $\varphi$ is a morphism from $X$ to $X^{\prime}$, and $\alpha$ is a 2-morphism from $f$ to $f^{\prime} \circ \varphi$. The full subcategory consisting of all $(X, f)$ such that $f$ is projective is called the category of projective $Y$-stacks.

Definition 2.1.2. (i) An inclusion of components is a morphism $f: X \rightarrow X^{\prime}$ which is an isomorphism of $X$ onto a union of connected components of $X^{\prime}$. If $Y$ is a stack, $(X, f)$ and $\left(X^{\prime}, f^{\prime}\right)$ are $Y$-stacks, and $g$ is a morphism from $(X, f)$ to $\left(X^{\prime}, f^{\prime}\right)$ in the category of $Y$-stacks (in short, a $Y$-morphism), then we say that $g$ is a $Y$-inclusion of components. The set of isomorphism classes of $Y$-stacks forms a directed set with $(X, f) \preceq\left(X^{\prime}, f^{\prime}\right)$ whenever there exists a morphism from $(X, f)$ to $\left(X^{\prime}, f^{\prime}\right)$ which is a $Y$-inclusion of components.

(ii) Let $Y$ be a stack. We denote by $\mathfrak{A}_{Y}$ the set of isomorphism classes of projective $Y$-stacks with the partial ordering of (i). For each $Y, \mathfrak{A}_{Y}$ is a directed set.

Definition 2.1.3. Let $Y$ be a stack. We denote by $\mathfrak{B}_{Y}$ the set of isomorphism classes of vector bundles over $Y$, partially ordered by declaring $E \preceq F$ whenever there exists a surjection of vector bundles $F \rightarrow E$.

Definition 2.1.4. (i) For $Y$ a stack, $Z_{*} Y$ denotes the group of algebraic cycles on $Y$, i.e., the free abelian group on the set of integral closed substacks of $Y$, graded by dimension [Gi1, V2]. We denote by $W_{*} Y$ the group of rational equivalences 
on $Y$. If $k(Z)^{*}$ denotes the multiplicative group of rational functions on an integral substack $Z$, not identically zero, then $W_{j} Y$ is the direct sum of $k(Z)^{*}$ over integral closed substacks $Z$ of dimension $j+1$. There is a map $\partial: W_{j} Y \rightarrow$ $Z_{j} Y$ which locally for the smooth topology sends a rational function to the corresponding Weil divisor.

(ii) The naïve Chow groups of $Y$ are defined to be $A_{k}^{\circ} Y=Z_{k} Y / \partial W_{k} Y$.

(iii) The Edidin-Graham-Totaro Chow groups ${ }^{1}$ [E-G1] are defined, for $Y$ connected, by $\widehat{A}_{k} Y=\lim _{\mathfrak{B}_{Y}} A_{k+\mathrm{rk} E}^{\circ} E$, and for $Y=Y_{1} \amalg \cdots \amalg Y_{r}$ with each $Y_{i}$ connected, by $\widehat{A}_{k} Y=\vec{\bigoplus}_{i=1}^{r} \widehat{A}_{k} Y_{i}$.

(iv) Given a morphism $f: X \rightarrow Y$ with $X$ connected, we define the restricted EdidinGraham-Totaro Chow groups to be the groups $\widehat{A}_{k}^{f} X=\lim _{\mathfrak{B}_{Y}} A_{k+\mathrm{rk} E}^{\circ} f^{*} E$. If $X=X_{1} \amalg \cdots \amalg X_{r}$ with each $X_{i}$ connected, we set $\widehat{A}_{k}^{f} X=\bigoplus_{i=1}^{r} \widehat{A}_{k}^{f} X_{i}$. There is a natural map $\iota_{f}: \widehat{A}_{k}^{f} X \rightarrow \widehat{A}_{k} X$.

(v) If $f: X \rightarrow Y$ is a projective morphism, the restricted projective pushforward is the map $f_{*}: \widehat{A}_{k}^{f} X \rightarrow \widehat{A}_{k} Y$ defined by each $\tilde{f}_{*}: A_{k}^{\circ} f^{*} E \rightarrow A_{k}^{\circ} E$ (for every $E$ we are letting $\tilde{f}: f^{*} E \rightarrow E$ denote the pullback of $f$ ).

Remark 2.1.5. If $E$ and $F$ are vector bundles on a stack $Y$, then any two vector bundle surjections $\varphi, \psi: E \rightarrow F$ induce the same map on naïve Chow groups. Indeed, let $\Psi_{t}=\varphi+t(\psi-\varphi)$ for $t$ in the base field. This defines a morphism $\Psi: E \times \mathbb{A}^{1} \rightarrow F$. Given any closed integral substack $Z$ of $F$, the closure $\overline{\Psi^{-1}(Z)}$ of $\Psi^{-1}(Z)$ in $E \times \mathbb{P}^{1}$ exhibits a rational equivalence between $\varphi^{*}[Z]$ and $\psi^{*}[Z]$ (the rational equivalence between the fiber over $t=0$ and the fiber over $t=1$ pushes forward to $E$ ). Thus $\left\{A_{r+\mathrm{rk} E}^{\circ} E\right\}$ forms a direct system of abelian groups over $\mathfrak{B}_{Y}$.

Remark 2.1.6. The natural map $A_{*}^{\circ} Y \rightarrow \widehat{A}_{*} Y$ is an isomorphism for any scheme $Y$ $[\mathrm{F}$, Theorem $3.3,(\mathrm{a})]$, or more generally for any algebraic space $[\mathrm{Kn}] Y$ (any algebraic space has a dense open subspace represented by a scheme, so projection from a vector bundle induces a pullback map on $A_{*}^{\circ}$ which is surjective by the argument of [F, Proposition 1.9], and injectivity is demonstrated exactly as for schemes).

Remark 2.1.7. The groups $\widehat{A}_{*} Y$ are defined in [E-G1] only in the special case of a quotient stack $Y \simeq[X / G]$ (with $G$ an algebraic group acting on an algebraic space $X$; the authors employ the notation $A_{*}^{G} X$ but point out that these groups are in fact invariants of the underlying stack $Y$ ). Because quotient stacks admit suitable approximations by algebraic spaces, the limit in Definition 2.1.4 (iii) stabilizes after some point, and now because the pullback map on Chow groups induced by a vector bundle over an algebraic space is an isomorphism, the content of Remark 2.1.5 becomes trivial and therefore not an ingredient in the construction of [E-G1].

\footnotetext{
${ }^{1}$ In [E-G1] the authors attribute the idea behind their construction to Burt Totaro.
} 
Remark 2.1.8. An inclusion of components gives rise to unrestricted projective pushforward, i.e., if $f: X \rightarrow Y$ is an inclusion of components then $\iota_{f}$ is an isomorphism.

Definition 2.1.9. Let $X$ be a stack. If $T$ is a stack, and $p_{1}$ and $p_{2}$ are projective morphisms $T \rightarrow X$, then the set

$$
\left\{p_{2 *} \beta_{2}-p_{1 *} \beta_{1} \mid\left(\beta_{1}, \beta_{2}\right) \in \widehat{A}_{k}^{p_{1}} T \oplus \widehat{A}_{k}^{p_{2}} T \text { satisfies } \iota_{p_{1}}\left(\beta_{1}\right)=\iota_{p_{2}}\left(\beta_{2}\right)\right\}
$$

is a subgroup of $\widehat{A}_{k} X$ which we denote $\widehat{B}_{k}^{p_{1}, p_{2}} X$. If $(X, f)$ is a $Y$-stack, the union of the subgroups $\widehat{B}_{k}^{p_{1}, p_{2}} X$, over all $T$ and all pairs of projective morphisms $p_{1}$ and $p_{2}$ such that $f \circ p_{1}$ is 2-isomorphic to $f \circ p_{2}$, is a subgroup of $\widehat{A}_{k} X$ which we denote $\widehat{B}_{k} X$.

Remark 2.1.10. Let $Y$ be a stack, and suppose we are given two $Y$-inclusions of components $f_{1}, f_{2}: X \rightarrow X^{\prime}$. Then for all $\alpha \in \widehat{A}_{*} X$, we have $f_{2 *} \alpha-f_{1 *} \alpha \in \widehat{B}_{k}^{f_{1}, f_{2}} X$ $\left(\right.$ take $\left.\beta_{1}=\beta_{2}=\alpha\right)$.

Definition 2.1.11. Let $Y$ be a stack. We define

$$
A_{k} Y=\lim _{\mathfrak{A}_{Y}}\left(\widehat{A}_{k} X / \widehat{B}_{k} X\right) .
$$

The main result of this preprint is

Theorem 2.1.12. Let $k$ be a base field. The functor $A_{*}$ of Definition 2.1.11, from the category of Artin stacks of finite type over $k$ to the category of abelian groups graded by dimension, is contravariant for morphisms which are flat of locally constant relative dimension, covariant for projective morphisms, and is related to the functors $A_{*}^{\circ}$ and $\widehat{A}_{*}$ via natural maps

$$
A_{*}^{\circ} X \rightarrow \widehat{A}_{*} X \rightarrow A_{*} X
$$

There is a canonically defined ring structure on $A_{*} X$ when $X$ is smooth and can be stratified by locally closed substacks which are each isomorphic to the quotient stack of an algebraic group acting on an algebraic space. The functor $A_{*}$ satisfies the following properties:

(i) For any algebraic space $X$, the map $A_{*}^{\circ} X \rightarrow A_{*} X$ is an isomorphism of groups, and for $X$ smooth is an isomorphism of rings.

(ii) For any Deligne-Mumford stack $X$, the map $A_{*}^{\circ} X \otimes \mathbb{Q} \rightarrow A_{*} X \otimes \mathbb{Q}$ is an isomorphism of groups, and for $X$ smooth is an isomorphism of rings.

(iii) For any algebraic space $X$ with action of a linear algebraic group $G$, the map $\widehat{A}_{*}[X / G] \rightarrow A_{*}[X / G]$ is an isomorphism of groups, and for $X$ smooth is an isomorphism of rings.

(iv) For any stack $X$ with closed substack $Z$ and complement $U$, the excision sequence $A_{j} Z \rightarrow A_{j} X \rightarrow A_{j} U \rightarrow 0$ is exact. 
(v) We have $A_{j} X=0$ for all $j>\operatorname{dim} X$.

(vi) If $\pi: E \rightarrow X$ is a vector bundle of rank $e$, then the induced pullback map $\pi^{*}: A_{j} X \rightarrow A_{j+e} E$ is an isomorphism.

(vii) If $\pi: E \rightarrow X$ is a vector bundle of rank $e$ with associated projective bundle $p: P(E) \rightarrow X$ and line bundle $\mathcal{O}_{E}(1)$ on $P(E)$, then the map

$$
\theta_{E}: \bigoplus_{i=0}^{e-1} A_{j-e+i+1} X \rightarrow A_{j} P(E)
$$

given by $\left(\alpha_{i}\right) \mapsto \sum_{i=0}^{e-1} c_{1}\left(\mathcal{O}_{E}(1)\right)^{i} \cap p^{*} \alpha_{i}$ is an isomorphism.

(viii) There are Segre classes and Chern classes of vector bundles, and these satisfy the usual universal identities.

(ix) There are Gysin maps for regular immersions and regular local immersions, and these maps are functorial, commute with each other, and are compatible with flat pullback and projective pushforward.

(x) If $X$ can be stratified by locally closed substacks which are isomorphic to quotient stacks, then for any vector bundle stack $\pi: B \rightarrow X$ of virtual rank $e$, the induced pullback map $\pi^{*}: A_{j} X \rightarrow A_{j+e} B$ is an isomorphism.

(xi) Gysin maps exist for l.c.i. morphisms $f: X \rightarrow Y$ whenever $X$ can be stratified by quotient stacks, and these maps are functorial, commute with each other, and are compatible with flat pullback and projective pushforward.

Remark 2.1.13. As we shall see, the class of stacks which can be stratified by quotient stacks includes Deligne-Mumford stacks, and more generally stacks with quasifinite diagonal (Proposition 4.5.7). This class of stacks is stable under representable morphisms and formation of products (Proposition 4.5.5).

Notation 2.1.14. We denote a typical element of $A_{k} Y$ by $(f, \alpha)$ with $f: X \rightarrow Y$ projective and $\alpha \in \widehat{A}_{k} X$. When we speak of a cycle we refer to such a choice among all the representatives of a given cycle class. Unless specifically stated to the contrary, an identity of cycles refers to an identity in $\widehat{A}_{*}$ of the relevant stack.

Remark 2.1.15. Explicitly, now, if $(X, f)$ is a projective $Y$-stack, an element $(f, \alpha)$ of $A_{k} Y$ is equivalent to zero if and only if there exists a $Y$-inclusion of components $i: X \rightarrow X^{\prime}$ for some projective $Y$-stack $\left(X^{\prime}, f^{\prime}\right)$ such that there exist projective morphisms $p_{1}, p_{2}: T \rightarrow X^{\prime}$ and $\beta_{i} \in A_{k}^{p_{i}} T(i=1,2)$ such that $f^{\prime} \circ p_{1}$ is 2-isomorphic to $f^{\prime} \circ p_{2}$, we have $\iota_{p_{1}}\left(\beta_{1}\right)=\iota_{p_{2}}\left(\beta_{2}\right)$ in $\widehat{A}_{*} T$, and $i_{*} \alpha=p_{2 *} \beta_{2}-p_{1 *} \beta_{1}$ in $\widehat{A}_{*} X^{\prime}$.

Remark 2.1.16. Suppose $f: X \rightarrow Y$ and $p_{1}, p_{2}: T \rightarrow X$ are projective and $g:=f \circ p_{1}$ and $f \circ p_{2}$ are 2-isomorphic. Then, for any $\beta_{1} \in \widehat{A}_{*}^{p_{1}} T$ and $\beta_{2} \in \widehat{A}_{*}^{p_{2}} T$, we have $\left(f, p_{2 *} \beta_{2}-p_{1 *} \beta_{1}\right)=\left(g, \iota_{p_{2}}\left(\beta_{2}\right)-\iota_{p_{1}}\left(\beta_{1}\right)\right)$ in $A_{*} Y$ (consider $q_{1}, q_{2}: T \amalg T \rightarrow X \amalg T$ 
given by $q_{1}=p_{1} \amalg 1_{T}$ and $\left.q_{2}=1_{T} \amalg p_{2}\right)$. In particular, $\left(g, \iota_{p_{1}}\left(\beta_{1}\right)\right)=\left(f, p_{1 *} \beta\right)$ in $A_{*} Y$. This plus Remarks 2.1.6 and 2.1.15 establishes that for any algebraic space $Y$, the natural map $A_{*}^{\circ} Y \rightarrow A_{*} Y$ is an isomorphism. Similarly, from the fact that whenever $\pi: E \rightarrow X$ is a vector bundle over a Deligne-Mumford stack $X$, the pullback map $\pi^{*}$ induces an isomorphism $A_{j}^{\circ} X \otimes \mathbb{Q} \rightarrow A_{j+\mathrm{rk} E}^{\circ} E \otimes \mathbb{Q}$, we conclude that the natural map $A_{*}^{\circ} Y \otimes \mathbb{Q} \rightarrow A_{*} Y \otimes \mathbb{Q}$ is an isomorphism.

Remark 2.1.17. Let a linear algebraic group $G$ act on an algebraic space $V$, and let $Y=[V / G]$ be the stack quotient of $V$ under the action of $G$. Then $A_{j} Y$ is the equivariant Chow group $A_{j}^{G} V$ of [E-G1]. Indeed, suppose $f: X \rightarrow Y$ is projective and $E \rightarrow X$ is a vector bundle. We consider a $r$-dimensional representation of $G$ such that the corresponding action of $G$ on affine space is free off of some locus of codimension larger than $\operatorname{dim} X-j$ (such exists for suitable $r$ ). Such a representation corresponds to a vector bundle on $B G$; we let $F$ denote the pullback of this bundle to $Y$ and $F^{\prime}$ the pullback to $X$. Now the map $A_{j+r}^{\circ} F^{\prime} \rightarrow A_{j+r+\mathrm{rk} E}^{\circ} E \oplus F^{\prime}$ induced by pullback is an isomorphism, so the map $A_{j+\mathrm{rk} E}^{\circ} E \rightarrow \widehat{A}_{j} X$ factors through $A_{j+r}^{\circ} F^{\prime}$, and now for any $\alpha \in A_{j+\mathrm{rk} E}^{\circ} E$ if we let $\alpha^{\prime}$ denote the element of $A_{j+r}^{\circ} F^{\prime}$ determined by this factorization, then we have $(f, \alpha)=\left(1_{Y}, f_{*}^{\prime} \alpha^{\prime}\right)$, where $f^{\prime}: F^{\prime} \rightarrow F$ denote the pullback of $f$. The resulting maps $A_{j+\mathrm{rk} E}^{\circ} E \rightarrow A_{j}^{G} V$ determine a map $A_{j} Y \rightarrow A_{j}^{G} V$ which is then seen to be inverse to the natural map $A_{j}^{G} V \simeq \widehat{A}_{j} Y \rightarrow A_{j} Y$.

\subsection{Basic operations on Chow groups}

The basic operations we consider here are flat pullback, projective pushforward, and Gysin maps for principal effective Cartier divisors.

Given a flat morphism $f: Y^{\prime} \rightarrow Y$ of locally constant relative dimension (but not necessarily representable), we define a pullback operation $f^{*}$ as follows. First, we observe that if $h: X \rightarrow Y$ is projective and if we form the fiber diagram

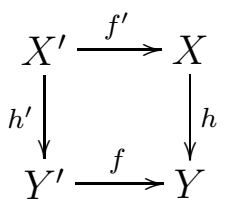

then $f^{\prime}$ is also flat of locally constant relative dimension, and if for any vector bundle $E \rightarrow X$ we denote by $\tilde{f}^{\prime}$ the pullback of $f^{\prime}$ via $E \rightarrow X$ then the maps $\tilde{f}^{\prime *}: A_{*}^{\circ} E \rightarrow$ $A_{*}^{\circ} E^{\prime}$ define a map $f^{\prime *}: \widehat{A}_{*} X \rightarrow \widehat{A}_{*} X^{\prime}$ (there is, of course, a shift in grading by the local relative dimensions of $f$ ). Since flat pullback on $A_{*}^{\circ}$ commutes with projective pushforward, the map $f^{\prime *}$ descends to give a map $\widehat{A}_{*} X / \widehat{B}_{*} X \rightarrow \widehat{A}_{*} X^{\prime} / \widehat{B}_{*} X^{\prime}$ and passes to the limit to give us

$$
f^{*}: A_{*} Y \rightarrow A_{*} Y^{\prime}
$$


As hinted in the introduction, the definition of proper pushforward is a tautology. Given a projective morphism $g: Y \rightarrow Z$, we define $g_{*}(f, \alpha)=(g \circ f, \alpha)$. By the remarks above, this map is compatible with restricted projective pushforward: $g_{*}\left(1_{Y}, \iota_{g}(\alpha)\right)=$ $\left(1_{Y}, g_{*} \alpha\right)$. More generally, suppose $h: W \rightarrow Z$ is projective. If we form the fiber square

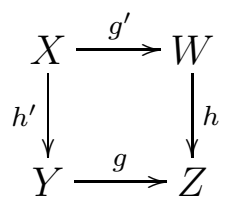

and if $\alpha \in \widehat{A}_{k}^{g^{\prime}} X$, then we have $g_{*}\left(h^{\prime}, \iota_{g^{\prime}}(\alpha)\right)=\left(h, g_{*}^{\prime} \alpha\right)$. In a fiber diagram, projective pushforward on $A_{*}$ commutes with flat pullback. This follows in a routine fashion from the similar fact for $A_{*}^{\circ}$.

Suppose $Y$ is a stack and we are given a morphism $\varphi: Y \rightarrow \mathbb{A}^{1}$. We denote by $Y_{0}$ the fiber of $\varphi$ over 0 and by $s$ the inclusion $Y_{0} \rightarrow Y$. There is a morphism $s^{*}: Z_{k} Y \rightarrow Z_{k-1} Y_{0}$ which sends $[V]$ to $\left[\left(\left.\varphi\right|_{V}\right)^{-1}(0)\right]$ if $V \not \subset Y_{0}$ and sends $[V]$ to 0 otherwise [F, Remark 2.3], as well as a compatible morphism $s^{*}: W_{k} Y \rightarrow W_{k-1} Y_{0}$ which sends a rational function $r$ on $V \not \subset Y_{0}$ to the formal sum of all the components of the tame symbol of $r$ and $\varphi$ which are supported in $Y_{0}$ [Gi3, Kr1]. Thus we obtain a map on rational equivalence classes of cycles $s^{*}: A_{k}^{\circ} Y \rightarrow A_{k-1}^{\circ} Y_{0}$. This map respects flat pullback, so we obtain $s^{*}: \widehat{A}_{k} Y \rightarrow \widehat{A}_{k-1} Y_{0}$.

By compatibility with proper pushforward we have the following form of the projection formula:

Proposition 2.2.1. Let $Y$ be a stack with morphism to $\mathbb{A}^{1}$ and let $f: X \rightarrow Y$ be a projective morphism. If we form the fiber diagram

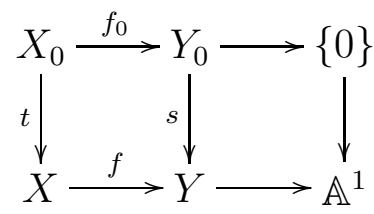

then $f_{0 *}\left(t^{*} \alpha\right)=s^{*}\left(f_{*} \alpha\right)$ for all $\alpha \in \widehat{A}_{k}^{f} X$ (where $f_{*}$ and $f_{0 *}$ denote restricted projective pushforward).

Given such a fiber diagram, the map $(f, \alpha) \mapsto\left(f_{0}, t^{*} \alpha\right)$ specifies the Gysin map $s^{*}: A_{k} Y \rightarrow A_{k-1} Y_{0}$. That this map respects equivalence is an application of Proposition 2.2.1, and is left to the reader. More generally, if $s: Y_{0} \rightarrow Y$ is a closed immersion and if there is a neighborhood $U$ of $Y_{0}$ and a function $\varphi: U \rightarrow \mathbb{A}^{1}$ such that $Y_{0}=U \times_{\mathbb{A}^{1}}\{0\}$, then the composite $A_{k} Y \rightarrow A_{k} U \rightarrow A_{k-1} Y_{0}$ is independent of $U$ and we call this map the Gysin map $s^{*}$. For instance, a morphism $Y \rightarrow \mathbb{P}^{1}$ determines a Gysin map $A_{k} Y \rightarrow A_{k-1}\left(Y \times_{\mathbb{P}^{1}}\{0\}\right)$. 


\subsection{Results on sheaves and vector bundles}

This section collects several elementary facts.

Proposition 2.3.1. Let $\mathcal{E}$ be a coherent sheaf on an open substack $U$ of a stack $X$. Then there exists a coherent sheaf $\mathcal{E}^{\prime}$ on $X$ such that $\left.\mathcal{E}^{\prime}\right|_{U} \simeq \mathcal{E}$.

Proof. This is [L-MB, Corollary 8.6].

Corollary 2.3.2. Let $X$ be a stack and let $U$ be an open substack. Given a projective morphism $g: S \rightarrow U$ with $S$ reduced, there exists a fiber diagram

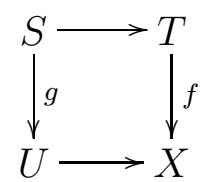

with $f$ projective.

Proposition 2.3.3. Let $X$ be a stack, with $U$ an open substack. Given any vector bundle $E \rightarrow U$, there exists a projective morphism $X^{\prime} \rightarrow X$ which is an isomorphism restricted to $U$, and a vector bundle $E^{\prime} \rightarrow X^{\prime}$, such that the restriction of $E^{\prime}$ to $U$ is isomorphic to E.

Proof. Let $r$ be the rank of $E$. Let $\mathcal{E}$ be the sheaf of sections of $E$. We may extend $\mathcal{E}$ to a coherent sheaf on all of $X$. If $X^{\prime} \rightarrow X$ is the Grassmannian of rank $r$ locally free quotients of $\mathcal{E}$ and $E^{\prime}$ is the universal quotient bundle, then $X^{\prime} \rightarrow X$ is an isomorphism over the locus where $\mathcal{E}$ is locally free (in particular, over $U$ ), and $E^{\prime}$ restricted to this locus agrees with the vector bundle determined by $\mathcal{E}$. There exists a closed immersion $X^{\prime} \rightarrow \mathbf{P}\left(\bigwedge^{r} \mathcal{E}\right)$ which exhibits $X^{\prime} \rightarrow X$ as a projective morphism.

Proposition 2.3.4. Let $X$ be a stack, and let $U$ be the complement of a Cartier divisor $D$ on $X$. Let $E$ and $F$ be vector bundles on $X$, and suppose an extension

$$
\left.\left.0 \rightarrow F\right|_{U} \rightarrow Q \rightarrow E\right|_{U} \rightarrow 0
$$

on $U$ is given. Then there exists an extension of $E$ by $F \otimes \mathcal{O}(n D)$ for some $n$ which restricts to the extension (2.3.1) on $U$.

Proof. We need only take $n$ sufficiently large so that the extension class of $Q$ lies in the image of $H^{1}\left(X, E^{*} \otimes F \otimes \mathcal{O}(n D)\right) \rightarrow H^{1}\left(U,\left.\left(E^{*} \otimes F\right)\right|_{U}\right)$.

In the statement of Proposition 2.3.4, the term "Cartier divisor" refers to a substack of codimension 1 which on some smooth atlas is given by the vanishing of a single function which is a non zero divisor. Since blowing up exhibits any open substack as the complement of a Cartier divisor, we have 
Corollary 2.3.5. Let $X$ be a stack, and let $U$ be an open substack. If vector bundles $E$ on $X$ and $F_{0}$ on $U$ and an extension $\left.0 \rightarrow F_{0} \rightarrow Q_{0} \rightarrow E\right|_{U} \rightarrow 0$ are given, then there exist a projective morphism $X^{\prime} \rightarrow X$ which is an isomorphism over $U$, a vector bundle $F$ on $X$ such that $\left.F\right|_{U} \simeq F_{0}$, and an extension $0 \rightarrow F \rightarrow Q \rightarrow E \rightarrow 0$ on $X^{\prime}$ whose restriction to $U$ is the given extension.

\subsection{The excision sequence}

Proposition 2.4.1. Let $Y$ be a closed substack of $X$ with inclusion map $\sigma$. Let $U$ be the complement of $Y$ in $X$, with $\rho: U \rightarrow X$. Then the sequence

$$
A_{*} Y \stackrel{\sigma_{*}}{\rightarrow} A_{*} X \stackrel{\rho^{*}}{\rightarrow} A_{*} U \rightarrow 0
$$

is exact.

Proof. For surjectivity on the right, suppose $(f, \alpha)$ is a cycle in $A_{*} U$, with $f: S \rightarrow U$ projective. By Corollary 2.3.2, $f$ is the restriction to $U$ of some projective morphism $T \rightarrow X$. If $\alpha$ is represented by a cycle in a vector bundle $E \rightarrow S$, then by Proposition 2.3.1, there exists $T^{\prime} \rightarrow T$ projective, which is an isomorphism over $S$, and a bundle $E^{\prime} \rightarrow T$ such that $\left.E^{\prime}\right|_{S} \simeq E$. Now the desired result follows since the restriction map $A_{*}^{\circ} E^{\prime} \rightarrow A_{*}^{\circ} E$ is surjective.

Since the composite $\rho^{*} \circ \sigma_{*}$ is clearly zero, all that remains is to show that any element of ker $\rho^{*}$ lies in the image of $\sigma_{*}$. By Remark 2.1.15 any element in the kernel of $\rho^{*}$ must have a representative of the form $(f, \alpha)$ where, if we let $g: S \rightarrow U$ denote the restriction of $f: T \rightarrow X$, there exist projective morphisms $p_{1}, p_{2}: V \rightarrow S$ for some $V$ such that $g \circ p_{1}$ is 2-isomorphic to $g \circ p_{2}$, and $\beta_{i} \in \widehat{A}_{*}^{p_{i}} V(i=1,2)$ such that $\iota_{p_{1}}\left(\beta_{1}\right)=\iota_{p_{2}}\left(\beta_{2}\right)$ and such that

$$
\rho^{\prime *} \alpha=p_{2 *} \beta_{2}-p_{1 *} \beta_{1}
$$

in $\widehat{A}_{*} S$ (where $\rho^{\prime}$ denotes the inclusion map $S \rightarrow T$ ).

Modifying $T$, we may assume the bundles on $S$ on whose pullbacks $\beta_{i}$ are defined are in fact restrictions of bundles $F_{i}$ on $T$. Moreover, we may assume (2.4.1) holds as a rational equivalence in a bundle which is the restriction of a bundle on $T$ (if there are surjections $\left.G \rightarrow F_{1}\right|_{S}$ and $\left.G \rightarrow F_{2}\right|_{S}$ then we form $\left.0 \rightarrow K \rightarrow G \oplus G \rightarrow\left(F_{1} \oplus F_{2}\right)\right|_{S} \rightarrow 0$ and apply Corollary 2.3.5). A choice of 2-isomorphism from $g \circ p_{1}$ to $g \circ p_{2}$ determines a morphism $V \rightarrow S \times_{U} S$, which must be projective, and by an application of Corollary 2.3.2 we may assume we have a fiber diagram

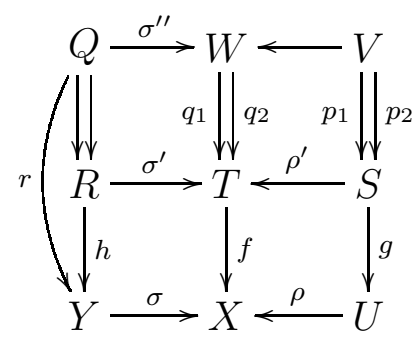


After a modification of $W$ we may assume the equality $\iota_{p_{1}}\left(\beta_{1}\right)=\iota_{p_{2}}\left(\beta_{2}\right)$ holds as a rational equivalence in a bundle which is the restriction of a bundle on $W$.

We may find $\gamma_{i} \in \widehat{A}_{*}^{q_{i}} W$, represented by a cycle in the bundle $q_{i}^{*} F_{i}$, which restricts to $\beta_{i}$, for $i=1,2$. By the excision axiom for naïve Chow groups, $\iota_{q_{2}}\left(\gamma_{2}\right)-\iota_{q_{1}}\left(\gamma_{1}\right)=\sigma_{*}^{\prime \prime} \varepsilon$ for some $\varepsilon \in \widehat{A}_{*}^{\sigma^{\prime \prime}} Q$, and then $\alpha=q_{2 *} \gamma_{2}-q_{1 *} \gamma_{1}+\sigma_{*}^{\prime} \delta$ for some $\delta \in \widehat{A}_{*}^{\sigma^{\prime}} R$. Now, by Remark 2.1.16,

$$
(f, \alpha)=\sigma_{*}(r, \varepsilon)+\sigma_{*}(h, \delta) .
$$

\section{Equivalence on bundles}

Convention 3.0.2. If $E$ is a vector bundle on a stack $X$, then $P(E)$ denotes the projectivization of $E$ in the sense of [F], i.e., $P(E)=\mathbf{P}\left(\operatorname{Sym}^{\bullet} \mathcal{E}^{*}\right)$, where $\mathcal{E}$ is the sheaf of sections of $E$. If $E$ and $F$ are vector bundles on a stack $X$, then $E \times_{X} F$ is also denoted using the Whitney sum notation $E \oplus F$.

Natural constructions in intersection theory produce cycles in vector bundles. An intersection theory requires, at the very least, a means of intersecting with the zero section of a vector bundle. This we develop by first examining trivial bundles (section 3.1), then developing a top Chern class operation (section 3.2), followed by a construction which deals with general cycles in vector bundles by compactifying and collapsing to the zero section (sections 3.3 and 3.4). After discussing affine bundles (section 3.5), we follow with some additional topics which form a standard part of intersection theory: Segre classes, Chern classes, and the projective bundle theorem (section 3.6).

On a scheme $X$, with a vector bundle $\pi: E \rightarrow X$ of rank $e$ and associated projective bundle $p: P(E) \rightarrow X$, the maps $\alpha \mapsto \pi^{*} \alpha$ and $\left(\alpha_{i}\right) \mapsto \sum_{i=0}^{e-1} c_{1}\left(\mathcal{O}_{E}(1)\right)^{i} \cap p^{*} \alpha_{i}$ are shown to be surjective using a noetherian induction argument which reduces the claim to the case of a trivial bundle; demonstrating injectivity takes more work and is proved first for projectivized bundles, and then for vector bundles ([F, §3]). The key fact that is used is that vector bundles on schemes are Zariski locally trivial. For stacks, the order of the steps is different: we first deduce injectivity of $\pi^{*}$ using the explicit formula for $\left(\pi^{*}\right)^{-1}[\mathrm{~F}$, Proposition 3.3]. Surjectivity is harder, and uses a particular equivalence of cycles on bundles (Proposition 3.4.1). The projective bundle theorem follows using the splitting principle (there is a pullback which transforms an arbitrary bundle into a filtered bundle) in a strong form (upon further pullback the filtration admits a splitting).

\subsection{Trivial bundles}

Let us start with a fact which is familiar from intersection theory on schemes. 
Proposition 3.1.1. Let $X$ be a stack, and $Z \subset X \times \mathbb{A}^{1}$ an integral closed substack. Let $\pi: X \times \mathbb{A}^{1} \rightarrow X$ be projection, with zero section s. Then $[Z]$ is rationally equivalent to $\pi^{*} s^{*}[Z]$ on $X \times \mathbb{A}^{1}$.

Proof. If $Z$ is contained in the fiber over zero then the claim is clear, so assume the contrary. Let $Z^{\prime}$ be the closure in $X \times \mathbb{A}^{1} \times \mathbb{P}^{1}$ of the substack $\left(1_{X} \times m\right)^{-1}(Z)$ of $X \times \mathbb{A}^{1} \times \mathbb{A}^{1}$, where $m$ is multiplication, $m(x, u)=u x$. If we think of $u$ as a time variable, then the fiber at time 1 is a copy of $Z$, and as $u$ approaches 0 , the cycle $Z$ is stretched towards infinity, until at time $u=0$, the fiber is identical to $\pi^{-1}\left(s^{-1}(Z)\right)$. Since $Z^{\prime} \rightarrow \mathbb{P}^{1}$ is flat away from infinity, the fibers over 0 and over 1 are rationally equivalent in $Z^{\prime}$. This rational equivalence pushes forward to a rational equivalence between $\pi^{*}\left[s^{-1}(Z)\right]$ and $[Z]$ on $X \times \mathbb{A}^{1}$.

From this, it is straightforward to deduce the corresponding result for $A_{*}$.

Proposition 3.1.2. Let $X$ be a stack, and let $\pi: X \times \mathbb{A}^{1} \rightarrow X$ be the projection map with zero section s. Then $\pi^{*}: A_{k} X \rightarrow A_{k+1} X \times \mathbb{A}^{1}$ is an isomorphism, with inverse $s^{*}$.

Proof. Clearly we have $s^{*} \circ \pi^{*}=$ id. It remains, therefore, to show that $\pi^{*}$ is surjective. By Proposition 2.4.1, it suffices to show that any cycle class on $X \times \mathbb{P}^{1}$ is represented by a cycle whose restriction to $X \times \mathbb{A}^{1}$ lies in the image of $\pi^{*}$. So, let $(f, \alpha)$ be a cycle on $X \times \mathbb{P}^{1}$, with $f: T \rightarrow X \times \mathbb{P}^{1}$ projective. We may assume $T$ is integral and $\alpha$ is represented by an integral closed substack $Z$ of the total space of a vector bundle $E \rightarrow T$. The cases where the composite $Z \rightarrow \mathbb{P}^{1}$ has image equal to $\{0\}$ or $\{\infty\}$ are easily dealt with, so we assume the contrary.

Let $Y=X \times \mathbb{P}^{1}$. Let $P$ be the closure in $\left(Y \times \mathbb{P}^{1}\right) \times_{X} Y$ of the graph $\Gamma$ of the morphism $1_{X} \times m: X \times \mathbb{A}^{1} \times \mathbb{A}^{1} \rightarrow X \times \mathbb{A}^{1}$ (where $m$ is as in Proposition 3.1.1). We have projections $\psi: P \rightarrow Y \times \mathbb{P}^{1}$ and $\varphi: P \rightarrow Y$. For $t$ in the base field, we may form the fiber diagram

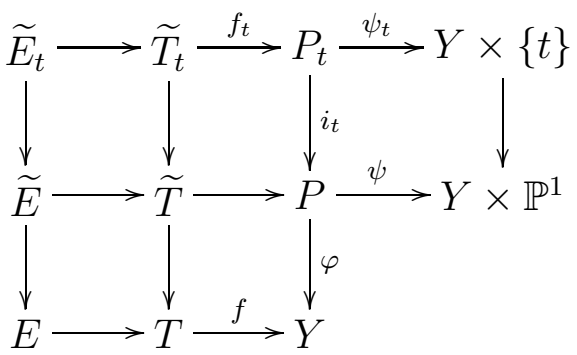

Although the map $\varphi$ is not flat, its restriction to $\Gamma$ is flat. If $\left[\left(\left.\varphi\right|_{\Gamma}\right)^{-1}(Z)\right]=$ $\sum a_{i}\left[W_{i}\right]$, we let $\tilde{\alpha}=\sum a_{i}\left[\overline{W_{i}}\right]$ and $\tilde{\alpha}_{t}=\sum a_{i}\left[\overline{W_{i}} \times_{\mathbb{P}^{1}}\{t\}\right]$. Then $\left(\psi_{0} \circ f_{0}, \tilde{\alpha}_{0}\right)=$ $\left(\psi_{1} \circ f_{1}, \tilde{\alpha}_{1}\right)$ in $A_{*} Y$. We now recognize that $\psi_{1}$ and $\varphi \circ i_{1}$ are isomorphisms, and that 
if we form the fiber square

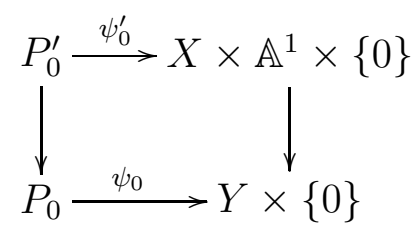

then $\psi_{0}^{\prime}$ is an isomorphism, and the composite $X \times \mathbb{A}^{1} \stackrel{\sim}{\rightarrow} P_{0}^{\prime} \rightarrow P_{0} \rightarrow P \rightarrow Y$ is equal to projection $X \times \mathbb{A}^{1} \rightarrow X$ followed by inclusion as the zero section $X \rightarrow Y$. Thus $\left(\psi_{0} \circ f_{0}, \tilde{\alpha}_{0}\right)$ restricts over $X \times \mathbb{A}^{1}$ to a pullback from $X$.

\subsection{The top Chern class operation}

Definition 3.2.1. (i) Let $X$ be a connected stack, and let $U \rightarrow X$ be a vector bundle of rank $r$. Then we have a map

$$
\widehat{A}_{j} X \rightarrow \widehat{A}_{j-r} X
$$

which, for any vector bundle $E \rightarrow X$, with $\operatorname{rk} E=s$, sends $\alpha \in A_{j+s}^{\circ} E$ to $s_{*} \alpha \in A_{j+s}^{\circ} E \oplus U$, where $s$ is the zero section of $E \oplus U \rightarrow E$. We denote the image class in $\widehat{A}_{j-r} X$ by $\alpha^{U}$. For $\alpha \in \widehat{A}_{*} X$ where $X$ is not connected, $\alpha^{U}$ denotes the sum of $\left(\left.\alpha\right|_{X_{i}}\right)^{U}$ over connected components $X_{i}$ of $X$.

(ii) Let $Y$ be a stack, and let $U \rightarrow Y$ be a vector bundle of rank $r$. We define the top Chern class operation

$$
c_{\text {top }}(U) \cap-: A_{j} Y \rightarrow A_{j-r} Y
$$

by $(f, \alpha) \mapsto\left(f, \alpha^{f^{*} U}\right)$. That this map respects equivalence is clear.

Proposition 3.2.2. Let $Y$ be a stack, $\pi: U \rightarrow Y$ a vector bundle, $f: T \rightarrow Y$ a projective morphism, $E \rightarrow T$ a vector bundle, and suppose $\gamma \in A_{*}^{\circ} E \oplus f^{*} U$. If we form the fiber diagram

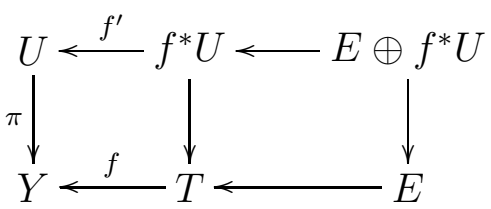

then $\pi^{*}(f, \gamma)=\left(f^{\prime}, \gamma\right)$, where on the right we view $\gamma$ as a cycle in the total space of the bundle $E \oplus f^{*} U \rightarrow f^{*} U$.

Proof. If $\tau$ denotes projection onto the first two factors $E \oplus f^{*} U \oplus f^{*} U \rightarrow E \oplus f^{*} U$ then $\pi^{*}(f, \gamma)=\left(f^{\prime}, \tau^{*} \gamma\right)$. But now we may let $v$ denote projection onto the first and third factors $E \oplus f^{*} U \oplus f^{*} U \rightarrow E \oplus f^{*} U$; by Remark 2.1.5, $\tau^{*} \gamma=v^{*} \gamma$ in $A_{*}^{\circ} E \oplus f^{*} U \oplus f^{*} U$, and we are done $\left(v^{*} \gamma \in A_{*}^{\circ} E \oplus f^{*} U \oplus f^{*} U\right.$ and $\gamma \in A_{*}^{\circ} E \oplus f^{*} U$ determine the same element of $\left.\widehat{A}_{*} f^{*} U\right)$. 
Corollary 3.2.3. Let $Y$ be a stack, and let $\pi: U \rightarrow Y$ be a vector bundle with zero section $s$. Then $\pi^{*} c_{\mathrm{top}}(U) \cap \alpha=s_{*} \alpha$ for all $\alpha \in A_{*} Y$.

The top Chern class operation obeys the expected properties.

Proposition 3.2.4. (i) If $E$ and $F$ are vector bundles on a stack $X$, then we have $c_{\text {top }}(E) \cap\left(c_{\text {top }}(F) \cap \alpha\right)=c_{\text {top }}(F) \cap\left(c_{\text {top }}(E) \cap \alpha\right)$ for all $\alpha \in A_{*} X$.

(ii) If $f: Y \rightarrow X$ is projective and $E$ is a vector bundle on $X$, then $f_{*}\left(c_{\mathrm{top}}\left(f^{*} E\right) \cap\right.$ $\alpha)=c_{\mathrm{top}}(E) \cap f_{*} \alpha$ for all $\alpha \in A_{*} X$.

(iii) If $f: Y \rightarrow X$ is flat of locally constant relative dimension and $E$ is a vector bundle on $X$, then $c_{\mathrm{top}}\left(f^{*} E\right) \cap f^{*} \alpha=f^{*}\left(c_{\mathrm{top}}(E) \cap \alpha\right)$ for all $\alpha \in A_{*} X$.

(iv) If $E$ is a vector bundle on a stack $X$ with a nowhere vanishing section s, then $c_{\text {top }}(E) \cap \alpha=0$ for all $\alpha \in A_{*} X$.

(v) If $L_{1}$ and $L_{2}$ are line bundles on a stack $X$, then $c_{\mathrm{top}}\left(L_{1}\right) \cap \alpha+c_{\mathrm{top}}\left(L_{2}\right) \cap \alpha=$ $c_{\text {top }}\left(L_{1} \otimes L_{2}\right) \cap \alpha$ for all $\alpha \in A_{*} X$.

(vi) If $L$ is a line bundle on a stack $X$, then $c_{\text {top }}(L) \cap \alpha+c_{\text {top }}\left(L^{\vee}\right) \cap \alpha=0$ for all $\alpha \in A_{*} X$.

Proof. Routine, for instance, the content of (v) is an explicit rational equivalence on $L_{1} \oplus L_{2} \oplus\left(L_{1} \otimes L_{2}\right)$, given locally by $(x, y, z) \mapsto x y z^{-1}$.

\subsection{Collapsing cycles to the zero section}

We present here a construction which is the reverse process of that of Section 3.1. Instead of stretching a cycle to infinity, we collapse a general cycle on a projectivized bundle $P(U \oplus 1)$ to the zero section, obtaining, away from the zero section, the pullback of the intersection with the boundary $P(U)$.

We first need some basic geometry of bundles and their projectivizations. Let $Y$ be a stack, with vector bundle $U$ of rank $r$, and consider the diagram as above:

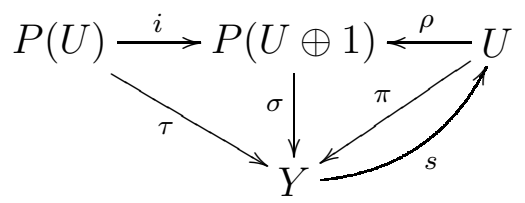

Let $s_{0}:=\rho \circ s$ be the zero section of $P(U \oplus 1) \rightarrow Y$, and let $\sigma_{0}:=s_{0} \circ \sigma$ be the map collapsing all of $P(U \oplus 1)$ onto the zero section. The map which projects $P(U \oplus 1)$ onto $P(U)$ is only a rational map, but becomes regular after blowing up the zero section. Denote the resulting projection morphism $\mathrm{B} \ell_{s_{0}(Y)} P(U \oplus 1) \rightarrow P(U)$ by $\eta$, and let $\xi$ be the blow-down. The maps $\eta$ and $\xi$ determine a closed immersion

$$
\mathrm{B} \ell_{s_{0}(Y)} P(U \oplus 1) \rightarrow P(U) \times_{Y} P(U \oplus 1)
$$


Proposition 3.3.1. With notation as above, let $f: T \rightarrow P(U \oplus 1)$ be projective and let $(f, \alpha)$ be a cycle on $P(U \oplus 1)$ represented by $[Z] \in A_{*}^{\circ} E$ for some vector bundle $E$ on $T$, with $Z$ integral and not supported over $P(U)$. Let $f^{\prime}: T^{\prime} \rightarrow P(U)$ be the restriction of $f$ and let $\alpha^{\prime} \in \widehat{A}_{*} T^{\prime}$ be given by $\left.\left[Z \times_{P(U \oplus 1)} P(U)\right] \in A_{*}^{\circ} E\right|_{T^{\prime}}$. Then

$$
(f, \alpha)=\xi_{*} \eta^{*}\left(f^{\prime}, \alpha^{\prime}\right)+\sigma_{0 *}(f, \alpha)
$$

in $A_{*} P(U \oplus 1)$.

Proof. Let $S=\mathrm{B} \ell_{s_{0}(Y) \times\{0\} \cup P(U) \times\{\infty\}} P(U \oplus 1) \times \mathbb{P}^{1}$. We have an inclusion

$$
S \hookrightarrow\left(P(U \oplus 1) \times \mathbb{P}^{1}\right) \times_{Y} P(U \oplus 1)
$$

whose image is given locally in coordinates as

$$
\begin{aligned}
& \left\{\left(\left(x_{1}: \ldots: x_{r}: t\right),(u: v)\right) \times\left(X_{1}: \ldots: X_{r}: T\right) \mid\right. \\
& \left.\quad\left(X_{1}, \ldots, X_{r}\right) \propto\left(x_{1}, \ldots, x_{r}\right) \text { and }\left(X_{1}, \ldots, X_{r}, T\right) \propto\left(u x_{1}, \ldots, u x_{r}, t v\right)\right\} .
\end{aligned}
$$

Let $\psi: S \rightarrow P(U \oplus 1) \times \mathbb{P}^{1}$ be the first projection; $\psi$ is the blow-down map. Let $\varphi$ be the second projection map.

The crucial properties of these maps are that away from $\infty=(0: 1)$ in $\mathbb{P}^{1}$, the map $\varphi$ is flat, and that the fiber of $S$ over $0=(1: 0) \in \mathbb{P}^{1}$ is isomorphic to $\left(\mathrm{B} \ell_{s_{0}(Y)} P(U \oplus 1)\right) \cup P(U \oplus 1)$, where the first component is blown down by $\psi$ onto $P(U \oplus 1) \times\{0\}$ and mapped by $\varphi$ via the projection map $\eta$ above, and the second component is collapsed to the zero section by $\psi$ and mapped isomorphically by $\varphi$. As in section 3.1 we think of $(u: v)$ as a time variable, and at time $1=(1: 1) \in \mathbb{P}^{1}$, both $\psi$ and $\varphi$ restrict to isomorphisms with $P(U \oplus 1)$.

The idea, expressed in the following diagram, is that the operation of pulling back a cycle on $P(U \oplus 1)$ via $\varphi$ and projecting by $\psi$ produces the pullback to $P(U \oplus 1)$ of the intersection of the cycle with the boundary $P(U) \subset P(U \oplus 1)$, plus a copy of the projection of the cycle to the zero section. We consider the fiber diagram

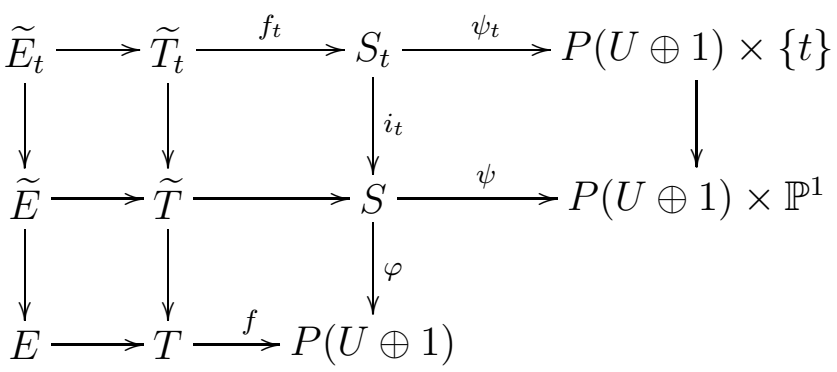

and then proceeding just as in the proof of Proposition 3.1.2, we deduce

$$
\left(\psi_{1} \circ f_{1},\left[\widetilde{Z}_{1}\right] \in A_{*}^{\circ} \widetilde{E}_{1}\right)=\left(\psi_{0} \circ f_{0},\left[\widetilde{Z}_{0}\right] \in A_{*}^{\circ} \widetilde{E}_{0}\right)
$$

in $A_{*} P(U \oplus 1)$, where $\widetilde{Z}_{t}=\varphi^{-1}(Z) \times_{\mathbb{P}^{1}}\{t\}$. The left- and right-hand sides of (3.3.3) are equal to the left- and right-hand sides of (3.3.2), respectively. 


\subsection{Intersecting cycles with the zero section}

We finally give a proof that the pullback map $\pi: U \rightarrow X$ for a vector bundle induces an isomorphism on $A_{*}$. This is achieved by giving an explicit inverse to $\pi^{*}$. We continue to use the notation of the previous section: $\pi: U \rightarrow Y$ is a vector bundle, with morphisms $\sigma, \tau$, etc. as before.

Proposition 3.4.1. Let $R$ be the universal quotient bundle of $\tau^{*} U$ on $P(U)$. Then for any $\alpha \in A_{*} P(U), \pi^{*} \tau_{*} c_{\text {top }}(R) \cap \alpha=\rho^{*} \xi_{*} \eta^{*} \alpha$ in $A_{*} U$.

Proof. Let $\alpha$ be represented by $(f,[Z])$, where $f: T \rightarrow P(U)$ is projective and $Z$ is an integral closed substack of $E$ for some vector bundle $E \rightarrow T$. Form the fiber diagram

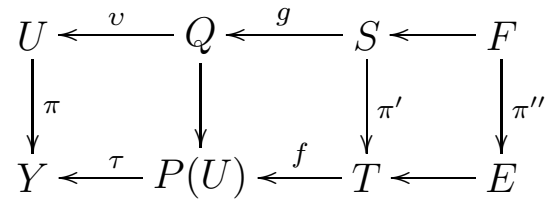

By the exact sequence of bundles on $P(U)$

$$
0 \rightarrow \mathcal{O}_{U}(-1) \rightarrow \tau^{*} U \rightarrow R \rightarrow 0
$$

we have

$$
c_{\mathrm{top}}(R) \cap \alpha=\left(f,\left[Z \times_{T} f^{*} \mathcal{O}_{U}(-1)\right] \in A_{*}^{\circ} F\right) .
$$

Then

$$
\tau_{*} c_{\text {top }}(R) \cap \alpha=\left(\tau \circ f,\left[Z \times_{T} f^{*} \mathcal{O}_{U}(-1)\right] \in A_{*}^{\circ} F\right) .
$$

Now by Proposition 3.2.2 we have

$$
\pi^{*} \tau_{*} c_{\text {top }}(R) \cap \alpha=\left(v \circ g,\left[Z \times_{T} f^{*} \mathcal{O}_{U}(-1)\right] \in A_{*}^{\circ} F\right)
$$

and this last expression we recognize as being a representative for $\rho^{*} \xi_{*} \eta^{*} \alpha$.

Proposition 3.4.2. Let $Q$ be the universal quotient bundle of $\sigma^{*}(U \oplus 1)$ on $P(U \oplus 1)$. Then

(i) for any $\alpha \in A_{*} P(U), c_{\mathrm{top}}(Q) \cap i_{*} \alpha=0$;

(ii) for any $\alpha \in A_{*} P(U \oplus 1), \pi^{*} \sigma_{*} c_{\text {top }}(Q) \cap \alpha=\rho^{*} \alpha$.

Proof. Let $f: T \rightarrow P(U \oplus 1)$ be projective, let $E \rightarrow T$ be a vector bundle, let $Z$ be an integral closed substack of $E$, and let $\alpha \in \widehat{A}_{*} T$ be given by $[Z] \in A_{*}^{\circ} E$.

By the exact sequence of bundles on $P(U \oplus 1)$

$$
0 \rightarrow \mathcal{O}_{U \oplus 1}(-1) \rightarrow \sigma^{*}(U \oplus 1) \rightarrow Q \rightarrow 0
$$


$\alpha^{f^{*} Q} \in \widehat{A}_{*} T$ is represented by $\left[Z \times_{T} f^{*} \mathcal{O}_{U \oplus 1}(-1)\right] \in A_{*}^{\circ} E \oplus f^{*} \sigma^{*} U \oplus 1$. If we consider $E \oplus f^{*} \sigma^{*} U \oplus 1 \rightarrow E \oplus f^{*} \sigma^{*} U$ as a trivial $\mathbb{A}^{1}$-bundle with zero section $t$ then by Proposition 3.1.1, $\alpha^{f^{*} Q}$ is also represented by $t^{*}\left[Z \times_{T} f^{*} \mathcal{O}_{U \oplus 1}(-1)\right] \in A_{*}^{\circ} E \oplus f^{*} \sigma^{*} U$. The intersection of $\mathcal{O}_{U \oplus 1}(-1)$ with the zero section of $\sigma^{*} U \oplus 1 \rightarrow \sigma^{*} U$ is the union of $\left.\mathcal{O}_{U \oplus 1}(-1)\right|_{P(U)}\left(=\mathcal{O}_{U}(-1)\right)$ and the zero section of $\mathcal{O}_{U \oplus 1}(-1)$. Statement (i) follows since $t^{*}$ is defined to be zero on cycles contained in the support of the divisor.

In the remaining case, that is, when the projection of $Z$ to $P(U \oplus 1)$ does not factor through $P(U)$, let $\left(f^{\prime}, \alpha^{\prime}:=\left.\left[Z^{\prime}\right] \in A_{*}^{\circ} E\right|_{T^{\prime}}\right)$ be determined from $\left(f, \alpha:=[Z] \in A_{*}^{\circ} E\right)$ as in the statement of Proposition 3.3.1. Then

$$
t^{*}\left[Z \times_{T} f^{*} \mathcal{O}_{U \oplus 1}(-1)\right]=\left[Z^{\prime} \times_{T^{\prime}} f^{\prime *} \mathcal{O}_{U}(-1)\right]+\tilde{s}_{*}[Z],
$$

in $A_{*}^{\circ} E \oplus f^{*} \sigma^{*} U$, where $\tilde{s}$ is the zero section of $E \oplus f^{*} \sigma^{*} U \rightarrow E$. Now, if we let $R$ be the universal quotient bundle of $\tau^{*} U$ on $P(U)$, then (3.4.1) implies the formula

$$
c_{\text {top }}(Q) \cap(f, \alpha)=i_{*} c_{\text {top }}(R) \cap\left(f^{\prime}, \alpha^{\prime}\right)+c_{\text {top }}\left(\sigma^{*} U\right) \cap(f, \alpha) .
$$

By Proposition 3.4.1 and the projection formula coupled with Corollary 3.2.3, we now have

$$
\pi^{*} \sigma_{*} c_{\text {top }}(Q) \cap(f, \alpha)=\rho^{*} \xi_{*} \eta^{*}\left(f^{\prime}, \alpha^{\prime}\right)+s_{*} \sigma_{*}(f, \alpha),
$$

and the desired statement follows by Proposition 3.3.1.

Proposition 3.4.3. Let $\pi: U \rightarrow Y$ be a vector bundle, with projectivization $\tau: P(U) \rightarrow$ $Y$. Let $R$ be the universal quotient bundle of $\tau^{*} U$. Then

$$
\tau_{*} c_{\mathrm{top}}(R) \cap \tau^{*} \alpha=\alpha
$$

for all $\alpha \in A_{*} Y$.

Proof. If $\alpha$ is represented by $\left(f,[Z] \in A_{*}^{\circ} E\right)$ where $f: T \rightarrow Y$ is projective and $E \rightarrow T$ is a vector bundle, and we form the fiber square

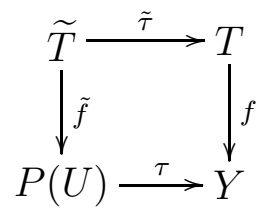

then $c_{\text {top }}(R) \cap \tau^{*} \alpha$ is represented by $\left[Z \times_{T} \tilde{f}^{*} \mathcal{O}_{U}(-1)\right] \in A_{*}^{\circ}\left(\tilde{\tau}^{*} E \oplus \tilde{\tau}^{*} f^{*} U\right)$. Since the bundle on which this cycle lives is the pullback of a bundle on $T$, we may compute $\tau_{*} c_{\text {top }}(R) \cap \tau^{*} \alpha$ by pushing forward the actual cycle. The push-forward map, restricted to $Z \times{ }_{\tilde{T}} \tilde{f}^{*} \mathcal{O}_{U}(-1)$, is a birational map, and hence $\tau_{*} c_{\text {top }}(R) \cap \tau^{*} \alpha$ is represented by $\left[Z \times_{T} f^{*} U\right] \in A_{*}^{\circ}\left(E \oplus f^{*} U\right)$, i.e., is equal to $\alpha$.

Corollary 3.4.4. The map $\alpha \mapsto \sigma_{*} c_{\mathrm{top}}(Q) \cap \bar{\alpha}$, where $\bar{\alpha} \in A_{*} P(U \oplus 1)$ is some cycle class that restricts to $\alpha$ (guaranteed to exist by excision), is independent of the choice of $\bar{\alpha}$ and gives an isomorphism $A_{*} U \rightarrow A_{*} Y$, and this map is inverse to the map $\pi^{*}$. 


\subsection{Affine bundles}

The structure group for affine $n$-plane bundles is the subgroup $A f f(n)$ of $G L(n+1)$ of matrices which have $n$ zeros followed by a 1 along the bottom row. Let $X$ be a stack; the map $\operatorname{Aff}(n) \rightarrow G L(n+1)$ associates to every locally trivial (for the smooth topology) affine $n$-plane bundle $\varphi: B \rightarrow X$ a vector bundle $E \rightarrow X$ of rank $(n+1)$, together with a surjection of vector bundles $\tau: E \rightarrow \mathcal{O}_{X}$. If $F$ denotes the kernel to $\tau$ then we have a closed immersion $P(F) \rightarrow P(E)$ with complement isomorphic to $B$. The affine bundle $\varphi: B \rightarrow X$ has the property that the associated surjection $\tau: E \rightarrow \mathcal{O}_{X}$ admits a splitting after pullback via $\varphi$.

Since a general affine bundle has no zero section, the formalism of section 3.3 does not apply, although we may still use the argument of section 3.4 to deduce that $\varphi^{*}$ is a split monomorphism. That $\varphi^{*}$ is a split monomorphism is one ingredient in the proof of the projective bundle theorem (section 3.6). Surjectivity of $\varphi^{*}$ comes only later as a corollary to the projective bundle theorem.

Lemma 3.5.1. Let $X$ be a scheme, and let $0 \rightarrow F \stackrel{i}{\rightarrow} E \rightarrow \mathcal{O}_{X} \rightarrow 0$ be an exact sequence of vector bundles on $X$. Then $i_{*} \alpha=0$ for all $\alpha \in A_{*} F$.

Proof. Denote by $\pi_{E}$ and $\pi_{F}$ the respective projections from $E$ and $F$. The Gysin map $i^{*}$ (from section 2.2) satisfies $i^{*} \circ \pi_{E}^{*}=\pi_{F}^{*}$, so by Corollary 3.4.4, $i^{*}$ is an isomorphism. By the definition of $i^{*}$ we have $i^{*} i_{*} \alpha=0$, so we conclude that $i_{*} \alpha=0$.

Proposition 3.5.2. Let $\varphi: B \rightarrow X$ be an affine n-plane bundle with associated vector bundle $E$ and exact sequence

$$
0 \rightarrow F \rightarrow E \rightarrow \mathcal{O}_{X} \rightarrow 0
$$

of vector bundles on $X$. Denote by $Q$ the universal quotient bundle of the pullback of $E$ via $\sigma: P(E) \rightarrow X$. Let $i$ denote the map $P(F) \rightarrow P(E)$. Then $c_{\text {top }}(Q) \cap i_{*} \alpha=0$ for all $\alpha \in A_{*} P(F)$.

Proof. Consider the pullback sequence $0 \rightarrow \sigma^{*} F \stackrel{i^{\prime}}{\rightarrow} \sigma^{*} E \rightarrow \mathcal{O}_{P(E)} \rightarrow 0$. With the diagram

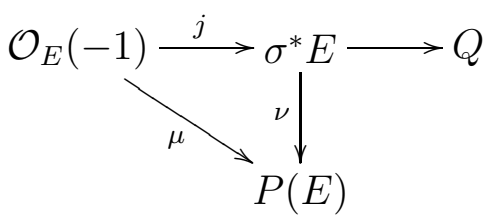

we have $\nu^{*}\left(c_{\text {top }}(Q) \cap i_{*} \alpha\right)=j_{*} \mu^{*} i_{*} \alpha=i_{*}^{\prime} k_{*} \psi^{*} \alpha$, where $\psi$ denote the projection $\mathcal{O}_{F}(-1) \rightarrow P(F)$ and $k$ denote the inclusion of $\mathcal{O}_{F}(-1)$ in $\sigma^{*} F$. But $i_{*}^{\prime} k_{*} \psi^{*} \alpha=0$ by Lemma 3.5.1, and $\nu^{*}$ is an isomorphism by Corollary 3.4.4, so we are done.

Now Proposition 3.4.3 gives us

Corollary 3.5.3. With notation as above, $\varphi^{*}$ is a split monomorphism. A splitting is the map sending $\alpha \in A_{*} B$ to $\sigma_{*}\left(c_{\mathrm{top}}(Q) \cap \bar{\alpha}\right)$, where $\bar{\alpha}$ is any cycle class on $P(E)$ which restricts to $\alpha$. 


\subsection{Segre and Chern classes and the projective bundle the- orem}

It is now routine to define general Segre classes and Chern classes of vector bundles. The usual projective bundle theorem will appear as a consequence.

Definition 3.6.1. Let $\pi: E \rightarrow X$ be a vector bundle on a stack $X$. The $i^{\text {th }}$ Segre class operation $s_{i}(E) \cap: A_{k} X \rightarrow A_{k-i} X$ is defined by the formula

$$
s_{i}(E) \cap \alpha=p_{*}\left(c_{\mathrm{top}}\left(\mathcal{O}_{E}(1)\right)^{\mathrm{rk} E-1+i} \cap p^{*} \alpha\right),
$$

where $p$ denotes the projection $P(E) \rightarrow X$.

Proposition 3.6.2. ([F, Proposition 3.1]) (i) Let $E$ be a vector bundle on a stack $X$. Then for all $\alpha \in A_{*} X$, we have $s_{i}(E) \cap \alpha=0$ for $i<0$ and $s_{0}(E) \cap \alpha=\alpha$.

(ii) If $E$ and $F$ are vector bundles on a stack $X$ and $\alpha \in A_{*} X$, then for all $i$ and $j, s_{i}(E) \cap\left(s_{j}(F) \cap \alpha\right)=s_{j}(F) \cap\left(s_{i}(E) \cap \alpha\right)$.

(iii) If $f: X^{\prime} \rightarrow X$ is projective, $E$ is a vector bundle on $X$, and $\alpha \in A_{*} X^{\prime}$, then, for all $i, f_{*}\left(s_{i}\left(f^{*} E\right) \cap \alpha\right)=s_{i}(E) \cap f_{*} \alpha$.

(iv) If $f: X^{\prime} \rightarrow X$ is flat of locally constant relative dimension, $E$ is a vector bundle on $X$, and $\alpha \in A_{*} X$, then, for all $i, s_{i}\left(f^{*} E\right) \cap f^{*} \alpha=f^{*}\left(s_{i}(E) \cap \alpha\right)$.

(v) If $E$ is a line bundle on $X$ and $\alpha \in A_{*} X$, then $s_{1}(E) \cap \alpha=-c_{\text {top }}(E) \cap \alpha$.

Proof. Only part (i) is nontrivial. By Corollary 3.4.4, it is enough to verify that the identities of part (i) hold after pullback to vector bundles. Consider the fiber diagram

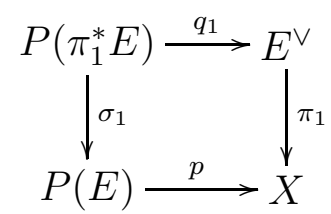

and the sequence of bundles

$$
0 \rightarrow S_{1} \stackrel{\iota}{\rightarrow} P\left(\pi_{1}^{*} E\right) \rightarrow \mathcal{O}_{E}(1) \rightarrow 0
$$

on $P\left(\pi_{1}^{*} E\right)$. Inductively, we define $\sigma_{k}, \pi_{k}$, and $q_{k}$ by the fiber diagram

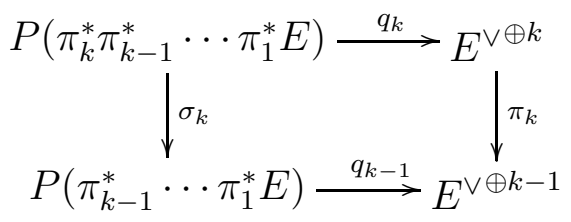


with

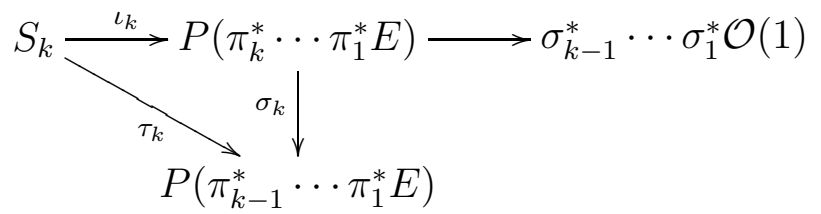

We have

$$
\pi_{k}^{*} \cdots \pi_{1}^{*}\left(c_{1} \mathcal{O}(1)^{k} \cap p^{*} \alpha\right)=q_{k *}\left(\iota_{k *} \tau_{k}^{*}\right) \cdots\left(\iota_{1 *} \tau_{1}^{*}\right) p^{*} \alpha .
$$

Set $e=\operatorname{rk} E-1$; it suffices to show that

$$
q_{k *}\left(\iota_{k *} \tau_{k}^{*}\right) \cdots\left(\iota_{1 *} \tau_{1}^{*}\right) p^{*} \alpha= \begin{cases}0 & \text { if } k<e \\ \pi_{k}^{*} \cdots \pi_{1}^{*} \alpha & \text { if } k=e\end{cases}
$$

What we do is show that the identity (3.6.1) holds for $Z_{*}$. This is a local computation, so we may assume $E$ is the trivial bundle of rank $e+1$. The cycle we are pushing forward along $X \times \mathbb{P}^{e} \times\left(\mathbb{A}^{e+1}\right)^{k} \rightarrow X \times\left(\mathbb{A}^{e+1}\right)^{k}$ has fiber equal to the set of points in $\mathbb{P}^{e}$ satisfying $k$ linear conditions, which are generically independent. Thus the generic fiber is positive-dimensional for $k<e$ and has degree 1 when $k=e$.

Proposition 3.6.3. Let $\pi: E \rightarrow X$ be a vector bundle on a stack $X$ with $\mathrm{rk} E=e+1$. Let $\sigma$ denote the projection $P(E) \rightarrow E$. Then the map $\theta_{E}: A_{*} X^{e+1} \rightarrow A_{*} P(E)$ given by $\left(\alpha_{0}, \ldots, \alpha_{e}\right) \mapsto \sum_{i=0}^{e} c_{1}\left(\mathcal{O}_{E}(1)\right)^{i} \cap \sigma^{*} \alpha_{i}$ is an isomorphism.

Proof. Injectivity is clear by Proposition 3.6.2. To demonstrate surjectivity of $\theta_{E}$, we induct on the rank of $E$. The case of a line bundle is trivial. For the inductive step, we first of all consider the special case where $E$ is filtered as $E \rightarrow L \rightarrow 0$, for some line bundle $L$ on $X$. It suffices to demonstrate that $\theta_{E \otimes L^{\vee}}$ is surjective (we have $\nu: P(E) \stackrel{\sim}{\rightarrow} P\left(E \otimes L^{\vee}\right)$ with $\left.\nu^{*} \mathcal{O}_{E \otimes L^{\vee}}(1)=\mathcal{O}_{E}(1) \otimes \sigma^{*} L\right)$, so we are reduced to the case where we have $E \rightarrow \mathcal{O}_{X} \rightarrow 0$. Then $E$ is associated to an affine bundle $\varphi: B \rightarrow X$, and we have seen that $\varphi^{*} E \rightarrow \mathcal{O}_{B} \rightarrow 0$ admits a splitting. Writing $\varphi^{*} E \simeq F \oplus 1$ we have $P(F) \rightarrow P(F \oplus 1)$ with complement $F$, so by the induction hypothesis and the exact sequence

$$
A_{*} P(F) \rightarrow A_{*} P(F \oplus 1) \rightarrow A_{*} F \rightarrow 0
$$

we obtain that $\theta_{\varphi^{*} E}$ is surjective. By Corollary 3.5.3 and Proposition 3.2.4 we conclude that $\theta_{E}$ is surjective.

We deduce the general case from the case above by the standard splitting construction: after pullback by $\tau: P\left(E^{\vee}\right) \rightarrow X$ we have $\tau^{*} E \rightarrow \mathcal{O}_{E^{\vee}}(1) \rightarrow 0$, and as before (this time using Proposition 3.4.3), we deduce surjectivity of $\theta_{E}$ from surjectivity of $\theta_{\tau^{*} E}$. 
We define Chern classes in terms of Segre classes using the universal polynomials. To deduce standard facts about Chern classes (vanishing of $c_{i}(E)$ for $i>\operatorname{rk} E$, projection formula, pullback formula, and Whitney sum formula) we use the splitting construction to reduce to the case of direct sums of line bundles, and then the formula $c_{1}(L)=c_{\text {top }}(L)$ (Proposition 3.6.2 (v)) implies the desired statements. By the same method, the formulas of [F, Remark 3.2.3] (Chern classes of dual bundles, tensor produces, etc.) hold as well for stacks; for instance we deduce the formula

$$
\zeta^{r}+c_{1}\left(p^{*} E\right) \zeta^{r-1}+\cdots+c_{r}\left(p^{*} E\right)=0
$$

which characterizes $A_{*} P(E)$, where $E$ is a vector bundle of rank $r$ on a stack $X$ with projection $p: P(E) \rightarrow X$ and with $\zeta=c_{1}\left(\mathcal{O}_{E}(1)\right)$ ([F, Remark 3.2.4]).

Let $\pi: E \rightarrow X$ be a vector bundle of rank $e$, with zero section $s$. The identity $\pi^{*} c_{\text {top }}(E) \cap \alpha=s_{*} \alpha$ (Corollary 3.2.3) which characterizes $c_{\text {top }}(E)$ also holds for $c_{e}(E)$, from which we deduce $c_{\text {top }}(E)=c_{e}(E)$, and so from now on we may use the two notations interchangeably.

Finally, the projective bundle theorem implies that the pullback map on Chow groups induced by an affine bundle is an isomorphism.

Corollary 3.6.4. Let $\varphi: B \rightarrow X$ be an affine bundle. Then $\varphi^{*}: A_{*} X \rightarrow A_{*} B$ is an isomorphism.

Proof. Let $E$ and $F$ be the associated vector bundles, as in section 3.5. Let $i$ denote the map $P(F) \rightarrow P(E)$, and let $p$ and $q$ denote the projection to $X$ from $P(E)$ and from $P(F)$, respectively. We observe that $\mathcal{O}_{E}(1)$ has a section vanishing precisely on $P(F)$, which implies $c_{1}\left(\mathcal{O}_{E}(1)\right) \cap p^{*} \alpha=i_{*} q^{*} \alpha$ and hence $c_{1}\left(\mathcal{O}_{E}(1)\right)^{j} \cap p^{*} \alpha=$ $i_{*} c_{1}\left(\mathcal{O}_{F}(1)\right)^{j-1} \cap q^{*} \alpha$ for $j \geq 1$. That $\varphi^{*}$ is an isomorphism now follows by the projective bundle theorem plus the excision axiom.

Remark 3.6.5. On a scheme, every affine bundle is locally trivial for the Zariski topology, so surjectivity of $\varphi^{*}$ follows by the elementary argument of $[\mathrm{F}, \S 1.9]$, and then injectivity can be proved using the same argument as for vector bundles (as remarked in $[\mathrm{Gr} 2]$, this argument uses only the existence of the compactification $P(E)$ ). More advanced techniques, cf. [Gi2], demonstrate homotopy invariance for any flat bundle on a scheme base such that the fibers over all points (closed or not) are isomorphic to affine spaces.

\section{Elementary intersection theory}

\subsection{Fulton-MacPherson construction for local immersions}

The existence of Gysin maps for principal effective Cartier divisors, together with the homotopy property (Corollary 3.4.4) and excision axiom, lets us apply the standard 
Fulton-MacPherson construction $[\mathrm{F}, \S 6]$ to produce Gysin maps for regular local immersions of Artin stacks. We recall that a representable morphism $f: F \rightarrow G$ of stacks is unramified if and only if, for some or equivalently every smooth atlas $V \rightarrow G$, the pullback $\tilde{f}: F \times_{G} V \rightarrow V$ fits into a commutative diagram

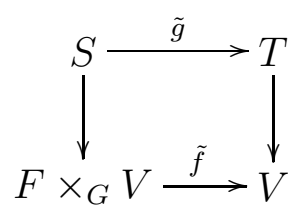

with $\tilde{g}$ a closed immersion and the vertical maps étale surjective ([EGA4, Corollary 18.4.8] plus the local nature of the property of being unramified). A representable morphism is called a local immersion if it is unramified, in which case there is a welldefined normal cone, given locally as the normal cone to $S \rightarrow T$. A representable morphism is called a regular local immersion if it is a local immersion and if moreover the map $\tilde{g}$ is a regular immersion (or equivalently, if the normal cone is a vector bundle).

Given a local immersion $f: F \rightarrow G$, and given an arbitrary morphism $g: G^{\prime} \rightarrow G$, there corresponds a map $A_{*} G^{\prime} \rightarrow A_{*}\left(C_{F} G \times_{F} F^{\prime}\right)$. If we set $F^{\prime}=F \times_{G} G^{\prime}$, then $f^{\prime}: F^{\prime} \rightarrow G^{\prime}$ is also a local immersion, and we may form the deformation space $M_{F^{\prime}}^{\circ} G^{\prime} \rightarrow \mathbb{P}^{1}$, which has general fiber $G^{\prime}$ and special fiber $s: C_{F^{\prime}} G^{\prime} \rightarrow M_{F^{\prime}}^{\circ} G^{\prime}$ (cf. [F, $\S 5.1]$ for the case of a closed immersion, and [Kr1] for the case of a local immersion). The map $A_{*} G \rightarrow A_{*}\left(C_{F} G \times{ }_{F} F^{\prime}\right)$ is defined to be the composite

$$
\begin{aligned}
A_{*} G^{\prime} & \rightarrow A_{*+1} G^{\prime} \times\left(\mathbb{P}^{1} \backslash\{0\}\right) \\
& \stackrel{\sim}{\rightarrow} A_{*+1} M_{F^{\prime}}^{\circ} G^{\prime} / A_{*+1} C_{F^{\prime}} G^{\prime} \stackrel{s^{*}}{\rightarrow} A_{*} C_{F^{\prime}} G^{\prime} \rightarrow A_{*}\left(C_{F} G \times{ }_{F} F^{\prime}\right)
\end{aligned}
$$

(the last map is pushforward via the closed immersion $C_{F^{\prime}} G^{\prime} \rightarrow C_{F} G \times{ }_{F} F^{\prime}$. In case $f: F \rightarrow G$ is a regular local immersion of codimension $d$, the cone $C_{F} G$ is a bundle, and we postcompose with the inverse to the pullback map $A_{*-d} F^{\prime} \stackrel{\sim}{\rightarrow} A_{*}\left(C_{F} G \times_{F} F^{\prime}\right)$ to obtain, finally, the refined Gysin homomorphism $f^{!}: A_{*} G^{\prime} \rightarrow A_{*-d} F^{\prime}$.

The refined Gysin map $f^{!}: A_{*} G^{\prime} \rightarrow A_{*-d} F^{\prime}$, on the level of actual cycles, amounts to nothing more than the usual refined Gysin map. If $T \rightarrow G^{\prime}$ is a projective morphism and $E \rightarrow T$ is a vector bundle, then applying $f^{!}$to the element of $A_{*} G^{\prime}$ given by $[V] \in A_{*}^{\circ} E$ yields the cycle class in $A_{*} F^{\prime}$ given by $f^{!}([V]) \in A_{*}^{\circ}\left(E \times_{F} G\right)$.

By purely formal arguments we see that the refined Gysin map to a regular local immersion is compatible with flat pullback and with projective pushforward. Functoriality of the refined Gysin map follows exactly as in [F, §6.5] (the claim for general $F \rightarrow G \rightarrow H$ reduces to the case when $H$ is a vector bundle over $G$ and $G \rightarrow H$ is the zero section, in which case the result follows from a local computation showing $\left.\left.N_{F} H \simeq N_{F} G \oplus H\right|_{F}\right)$. The argument for commutativity of refined Gysin homomorphisms corresponding to a pair of regular local immersions of stacks reduces by formal manipulations to the statement of [F, Theorem 6.4], and this we know by [Kr1, Proposition 4]. 


\subsection{Exterior product}

Let $X$ and $Y$ be stacks. Since the product of projective morphisms is again projective, a pair consisting of a cycle $(f, \alpha)$, with $f: S \rightarrow X$ and $\alpha \in \hat{A}_{*} S$, and a cycle $(g, \beta)$, with $g: T \rightarrow Y$ and $\beta \in \hat{A}_{*} T$, determines a cycle $(f \times g, \alpha \times \beta) \in A_{*}(X \times Y)$.

Proposition 4.2.1. The map, sending $((f, \alpha),(g, \beta))$ to $(f \times g, \alpha \times \beta)$, determines a morphism $A_{*} X \otimes A_{*} Y \rightarrow A_{*}(X \times Y)$.

The proof involves only routine checking of details.

\subsection{Intersections on Deligne-Mumford stacks}

Intersection theory on stacks was motivated by a desire to establish foundations for enumerative calculus on moduli spaces. A particularly enlightening early investigation in this direction is $[\mathrm{M}]$, where foundations are laid for constructing intersection rings with rational coefficients on certain moduli spaces of curves. The intersection ring of the compactified moduli space $\bar{M}_{2}$ of curves of genus 2 is described in detail, with the fractional coefficients that appear attributed to automorphisms of the curves. Later, more general, approaches to intersection theory on Deligne-Mumford stacks [Gi1, V2] also produce intersection operations and intersection products on the Chow groups with rational coefficients.

The functor $A_{*}$ allows the construction of an integer-valued intersection product. If $X$ is a smooth Deligne-Mumford stack, then the diagonal $X \rightarrow X \times X$ is a regular local immersion, so the contents of sections 4.1 and 4.2 yield an intersection product on $A_{*} X$. This intersection product satisfies the usual properties, cf. [F, §8.3]. In particular, it agrees with the intersection product of [E-G1] in case $X$ is a global quotient.

Even though we have an integer-valued intersection ring, we still need to tensor with $\mathbb{Q}$ if we wish to do enumerative geometry. If $X$ is a complete Deligne-Mumford stack over a field $k$, then the map $X \rightarrow$ Spec $k$ is proper (cf. [D-M] or [V2] for a definition) but non-representable, so we do not get a pushforward in the $A_{*}$ theory. What we have is a cycle map back to the naïve Chow groups

$$
\text { cyc }: A_{*} X \rightarrow A_{*} X \otimes \mathbb{Q} \stackrel{\sim}{\rightarrow} A_{*}^{\circ} X \otimes \mathbb{Q},
$$

which introduces denominators from the fact that the Gysin map for vector bundles is defined only rationally on $A_{*}^{\circ}$. Then, we have a pushforward on naïve Chow groups

$$
\int_{X}: A_{0}^{\circ} X \otimes \mathbb{Q} \rightarrow A_{0}^{\circ} \operatorname{Spec} k \otimes \mathbb{Q} \simeq \mathbb{Q},
$$

cf. [V2], and this pushforward may introduce even more denominators. As an example, the compactified moduli space $\bar{M}_{1,1}$ of elliptic curves over the complex numbers has two special points with stabilizer groups cyclic of orders 4 and 6 , respectively, and generic point with stabilizer group $\mathbb{Z} / 2$. For all $\alpha \in A_{0} \bar{M}_{1,1}$ we have 2 cyc $(\alpha) \in$ $\operatorname{im}\left(A_{0}^{\circ} \bar{M}_{1,1} \rightarrow A_{0} \bar{M}_{1,1}\right)$. However, if we let $\pi: U \rightarrow \bar{M}_{1,1}$ be the universal curve and let $E=\pi_{*} \omega_{U / \bar{M}_{1,1}}$ be the Hodge bundle, then we find $\int_{\bar{M}_{1,1}} c y c\left(c_{1}(E)\right)=1 / 24$. 


\subsection{Boundedness by dimension}

Since projective pushforward lowers codimension, there is the potential, a priori, that there can be nontrivial cycle classes in $A_{k} X$ for $k$ greater than the dimension of $X$. This turns out not to occur; the justification uses some facts about projective morphisms plus the splitting principle. We first prove a preliminary lemma, and then prove the vanishing of $A_{k} X$ for $k>\operatorname{dim} X$. This section, as well as our deduction of Corollary 2.3.2 from Proposition 2.3.1, make clear why we do not develop the pushforward for general proper morphisms, but only for projective morphisms.

Lemma 4.4.1. Let $Y$ be a stack of dimension d, let $E \rightarrow Y$ be a vector bundle of rank $r$ with projectivization $p: P(E) \rightarrow Y$, and let $U_{1}$ and $U_{2}$ be vector bundles on $Y$ of ranks $e_{1}$ and $e_{2}$, respectively. Suppose $\gamma \in A_{k}^{\circ}\left(\left(p^{*} U_{1}\right)(m) \oplus p^{*} U_{2}\right)$ with $k>d+e_{1}+e+2$ (here $\left(p^{*} U_{1}\right)(m)$ denotes $\left.\left(p^{*} U_{1}\right) \otimes \mathcal{O}_{E}(1)^{\otimes m}\right)$. Then $(p, \gamma)=0$ in $A_{*} Y$.

Proof. We induct on $r$. The case $r=1$ is trivial. For the inductive step, we consider $q: P:=P\left(E^{\vee}\right) \rightarrow Y$, with $q^{*} E \rightarrow \mathcal{O}_{E^{\vee}}(1) \rightarrow 0$ on $P$. If we let $L=\mathcal{O}_{E^{\vee}}(-1)$ then we have

$$
0 \rightarrow K \rightarrow q^{*} E \otimes L \rightarrow \mathcal{O}_{P} \rightarrow 0
$$

on $P$, for some $K$ of rank $(r-1)$. Let $E^{\prime}=p^{*} E \otimes L$. Then $\mathcal{O}_{E^{\prime}}(1)$ has a global section which vanishes precisely on $P(K) \subset P\left(E^{\prime}\right)$. Moreover we may identify $P\left(E^{\prime}\right)$ with $P\left(q^{*} E\right)$, i.e., $P\left(E^{\prime}\right)$ fits into a fiber diagram

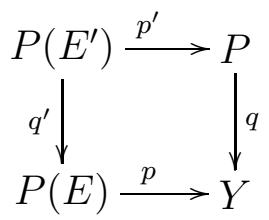

and moreover if we make this identification then we find $q^{*}\left(\left(p^{*} U_{1}\right)(m)\right) \simeq\left(p^{*} U_{1}^{\prime}\right) \otimes$ $\mathcal{O}_{E^{\prime}}(1)^{\otimes m}$, where $U_{1}^{\prime}=q^{*} U \otimes L^{\otimes m}$. If we set $U_{2}^{\prime}=q^{*} U_{2}$ then we have $q^{\prime *} \simeq p^{*} U_{2}^{\prime}$, so via these identifications we have

$$
q^{*}(p, \gamma)=\left(p^{\prime}, \delta\right)
$$

with $\delta \in A_{k+r-1}^{\circ}\left(\left(p^{*} U_{1}^{\prime}\right)(m) \oplus p^{*} U_{2}^{\prime}\right)$.

Since $\mathcal{O}_{E^{\prime}}(1)$ has a section nonvanishing on $P\left(E^{\prime}\right) \backslash P(K)$, we may find $\delta^{\prime} \in$ $A_{k+r-1}^{\circ}\left(p^{*} U_{1}^{\prime} \oplus p^{*} U_{2}^{\prime}\right)$ such that $\delta$ and $\delta^{\prime}$ have the same image under restriction to $A_{k+r-1}^{\circ}\left(u^{*} p^{\prime *} U_{1}^{\prime} \oplus u^{*} p^{\prime *} U_{2}^{\prime}\right)$, where $u: P\left(E^{\prime}\right) \backslash P(K) \rightarrow P\left(E^{\prime}\right)$ denotes inclusion. If we consider the projections

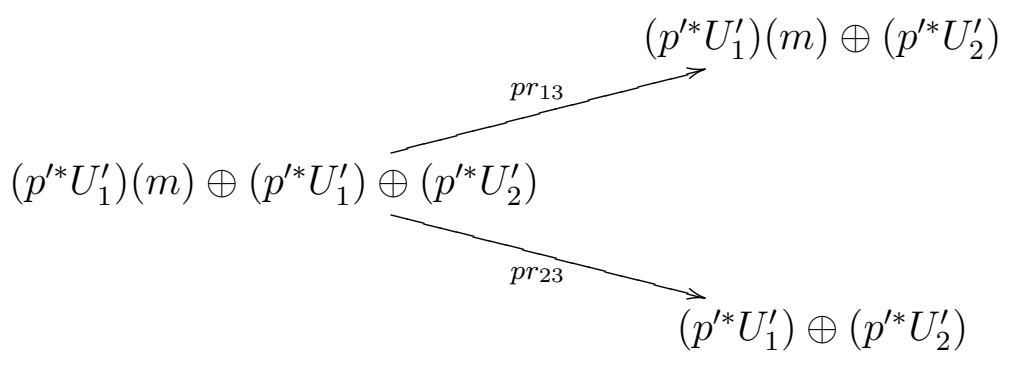


we have, by Remark 2.1.5 plus the standard excision sequence for naïve Chow groups,

$$
p r_{13}^{*} \delta=p r_{23}^{*} \delta^{\prime}+i_{*}^{\prime} \varepsilon
$$

for some $\varepsilon \in A_{k+r-1+e_{1}}^{\circ}\left(\left(i^{*} p^{\prime *} U_{1}^{\prime}\right)(m) \oplus\left(i^{*} p^{\prime *} U_{1}^{\prime}\right) \oplus\left(i^{*} p^{\prime *} U_{2}^{\prime}\right)\right)$, where $i: P(E) \rightarrow P\left(E^{\prime}\right)$ denotes inclusion and $i^{\prime}$ denotes the pullback of $i$. We find $\left(p^{\prime}, \delta^{\prime}\right)=0$ for dimension reasons, and $\left(p^{\prime} \circ i, \varepsilon\right)=0$ by the induction hypothesis, and hence $\left(p^{\prime}, \delta\right)=0$ in $A_{*} P$. Since $q^{*}$ is injective, we have $(p, \gamma)=0$ in $A_{*} Y$ as desired.

Proposition 4.4.2. Let $Y$ be a stack. We have $A_{k}(Y)=0$ for all $k>\operatorname{dim} Y$.

Proof. It suffices to show that for any $\alpha \in A_{k}(Y)$ that there exists a nonempty open substack $U$ of $Y$ such that, with inclusion map $\nu: U \rightarrow Y$, we have $\nu^{*} \alpha=0$ in $A_{k}(U)$. It suffices to consider $\alpha=(f, \gamma)$ with $f: T \rightarrow Y$ projective and $\gamma \in A_{*}^{\circ} F$ with $F$ a vector bundle over $T$. Shrinking $Y$, we may assume $f$ factors (up to 2-isomorphism) as a closed immersion followed by a projection of the form $P(E) \rightarrow Y$ where $E$ is a vector bundle on $Y$. There is a bundle $\mathcal{O}(1)$ on $P(E)$, hence also on $T$, and if we let $\mathcal{F}$ denote the sheaf of sections of $F$, then the natural map $f^{*} f_{*} \mathcal{F}(m) \rightarrow \mathcal{F}(m)$ is surjective for suitable $m$. Shrinking $Y$ further, we may suppose $f_{*} \mathcal{F}(m)$ is locally free, so we have a surjection of vector bundles $\left(f^{*} V\right)(-m) \rightarrow F$, and so we are reduced to the case $\alpha=(p, \gamma)$ with $p: P(E) \rightarrow Y$ the projection map and $\gamma \in A_{*}^{\circ}\left(\left(p^{*} V\right)(-m)\right)$ for $V$ a vector bundle on $Y$. But now $(p, \gamma=0)$ in $A_{*} Y$ by Lemma 4.4.1, so we are done.

\subsection{Stratifications by quotient stacks}

As promised in the introduction, we shall eventually show that whenever $\pi: B \rightarrow$ $Y$ is a vector bundle stack, such that the base $Y$ admits a stratification by global quotient stacks, then $\pi^{*}: A_{*} Y \rightarrow A_{*} B$ is an isomorphism. To prove this requires the localization machinery of section 5 . At this point, we content ourselves with some elementary observations, first describing classes of stacks which admit such stratifications and then showing (Proposition 4.5.8 (ii)) how to obtain the desired homotopy property when $Y$ is a suitable global quotient (this includes many cases of interest, e.g., certain moduli spaces).

Lemma 4.5.1. Let $X$ be an algebraic space, and let $G$ be a linear algebraic group acting on $X$. Then $[X / G]^{\text {red }}$ contains a nonempty open substack isomorphic to $[V / G L(n)]$ for some quasiprojective scheme $V$ with linear action of $G L(n)$.

Proof. Replacing $X$ by $X \times G L(n) / G$ we may assume $G=G L(n)$ for some $n$; then $[X / G]^{\text {red }}=\left[X^{\text {red }} / G\right]$, so we may as well assume $X$ reduced. Choosing an irreducible affine open $U \subset X$, we may replace $X$ by the image of the action map $U \times G \rightarrow X$, and now there must exist a finite subset $S=\left\{g_{i}\right\}$ of closed points of $G$ such that $U \times S \rightarrow X$ is surjective and such that the residue field of each $g_{i}$ is separable over the 
base field. So, for some finite separable field extension $k \rightarrow k^{\prime}$ of the base field, there is a cover of $X_{k^{\prime}}:=X \times_{\text {Spec } k}$ Spec $k^{\prime}$ by affine schemes, and hence $X_{k^{\prime}}$ is a scheme. By [S] there exists a quasiprojective $G$-stable dense open subscheme $V \subset X_{k^{\prime}}$. If $Z$ denotes the complement $X_{k^{\prime}} \backslash V$ then the image $Y$ of $Z$ under the finite map $X_{k^{\prime}} \rightarrow X$ is a proper closed subscheme of $X$, and now $X \backslash Y$ is $G$-stable and is a quasiprojective scheme (since $(X \backslash Y)_{k^{\prime}}$ is a quasiprojective scheme). Finally, now, $\left[(X \backslash Y)^{\text {reg }} / G\right]$ is a nonempty open substack of our original stack which is the quotient by $G=G L(n)$ of a quasiprojective scheme that is regular, and in particular normal, so the action of the group is linearizable.

Proposition 4.5.2. Let $Y$ be a stack. The following are equivalent.

(i) There exists a stratification of $Y^{\mathrm{red}}$ by locally closed substacks $U_{i}$ such that each $U_{i}$ is isomorphic to a stack of the form $\left[X_{i} / G_{i}\right]$, where for each $i, X_{i}$ is an algebraic space, and $G_{i}$ is a linear algebraic group acting on $T_{i}$;

(ii) There exists a stratification of $Y^{\mathrm{red}}$ by locally closed substacks $U_{i}$ such that each $U_{i}$ is isomorphic to a stack of the form $\left[T_{i} / G_{i}\right]$, where for each $i, T_{i}$ is a quasiprojective scheme and $G_{i}$ is a smooth connected linear algebraic group acting linearly on $T_{i}$.

Proof. Immediate from Lemma 4.5.1.

Definition 4.5.3. A stack $Y$ is said to admit a stratification by global quotients if the conditions of Proposition 4.5.2 are satisfied for $Y$.

Convention 4.5.4. The words global quotient or quotient stack, without any additional qualifyers, refer from now on to quotients of an algebraic space by an arbitrary linear algebraic group, as in (i) above.

Proposition 4.5.5. (i) Let $X$ and $Y$ be stacks which admit stratifications by global quotients. Then $X \times Y$ admits a stratification by global quotients.

(ii) Let $Y$ be a stack which admits a stratification by global quotients, and let $f: X \rightarrow Y$ be a representable morphism. Then $X$ admits a stratification by global quotients.

(iii) Every Deligne-Mumford stack admits a stratification by global quotients.

Proof. Since the product of global quotient stacks is again a global quotient stack, (i) is clear. Claim (ii) follows from the fact that for any algebraic group $G$, a representable morphism $U \rightarrow B G$ leads to an action of $G$ on $X:=U \times_{B G} \operatorname{Spec} k$ such that $U \simeq$ $[X / G]$. For (iii), if $f: U \rightarrow F$ is an étale presentation of a Deligne-Mumford stack $F$, then the restriction of $f$ over some nonempty open substack $G$ of $F$ is finite étale of some degree $n$, and $G$ is isomorphic to the quotient of the complement of all the diagonal components of $U \times{ }_{G} U \times{ }_{U} G \cdots \times{ }_{G} U$ ( $n$ copies) by the symmetric group $S^{n}$ (cf. [L-MB, (10.2)]). 
Proposition 4.5.6. Let $X$ be a stack. The following are equivalent.

(i) For every integer $N$, there exist a vector bundle $E \rightarrow X$ and a representable open substack $U$ of $E$ such that $E \backslash U$ has codimension $\geq N$ in $E$.

(ii) There exist a vector bundle $E \rightarrow X$ and a locally closed immersion $T \rightarrow E$, with $T$ representable and $T \rightarrow E$ surjective.

(iii) There exists an algebraic space $P$ with action of $G L(n)$ for some $n$, such that $X \simeq[P / G L(n)]$.

(iv) $X$ is a global quotient stack.

Proof. Conditions (iii) and (iv) are equivalent and, by the construction of [E-G1], imply (i). Clearly (i) implies (ii). Suppose (ii) holds, and let $P$ be the principal bundle associated to the vector bundle $\pi: E \rightarrow X$, so that $X \simeq[P / G L(n)]$, where $n=\operatorname{rk} E$. We claim $P$ must be representable.

We may assume $k$ is algebraically closed. We may also assume $T$ is disjoint from the zero section $s(X)$ (replace $T$ by $\left[T \cup \pi^{-1}(T \cap s(X))\right] \backslash s(X)$ ). Choosing, say, the first basis element of a framing yields a representable, faithfully flat morphism $P \rightarrow E \backslash s(X)$. The pre-image of $T$ is a representable, locally closed substack $S$ of $P$, such that the translates of $S$ by elements of $G L(n)$ cover the $k$-valued points of $P$. Hence $P$ is representable.

When the base field has positive characteristic, there exist stacks $Y$ which have finite stabilizer at every point but are not Deligne-Mumford.

Proposition 4.5.7. Let $Y$ be a stack. Then the following are equivalent.

(i) The diagonal map $Y \rightarrow Y \times Y$ is quasi-finite.

(ii) The stabilizer $Y \times_{Y \times Y} Y \rightarrow Y$ is quasi-finite.

Moreover, if $Y$ has quasi-finite diagonal then $Y$ admits a stratification by global quotients.

Proof. Clearly (i) implies (ii). For the converse, it suffices to check that whenever $\Omega$ is an algebraically closed field containing the base field $k$, then for any $x, y \in Y(\Omega)$ the set $\operatorname{Isom}_{Y}(x, y)(\Omega)$ is finite. But if $\operatorname{Isom}_{Y}(x, y)(\Omega)$ is nonempty, then for any $t \in \operatorname{Isom}_{Y}(x, y)(\Omega)$, postmultiplication with the inverse to $t$ gives an injective map $\operatorname{Isom}_{Y}(x, y) \rightarrow \operatorname{Isom}_{Y}(x, x)$, so (ii) implies (i) (cf. [D-M] or [V2] for notation). Now assume $Y$ has quasi-finite diagonal. We replace $Y$ by $T^{\text {red }}$ and let $f: U \rightarrow Y$ be a smooth presentation. By [L-MB, (5.7)], for a suitable closed subscheme $V$ of $U$, the map $V \rightarrow Y$ is dominant, and the restriction over a dense open substack of $Y$ is finite and flat. Let us shrink $Y$; if $E \rightarrow Y$ denotes the vector bundle whose sheaf of sections is $f_{*} \mathcal{O}_{V}$, then we have a closed immersion $V \rightarrow E$, so by Proposition 4.5.7, $Y$ is a global quotient. 
Proposition 4.5.8. Let $X$ be a quasiprojective scheme, let $G$ be a connected smooth linear algebraic group acting linearly on $X$, and let $Y=[X / G]$. Suppose $\pi: B \rightarrow Y$ is a vector bundle stack. Then

(i) B has vector bundles with total spaces represented by schemes off of loci of arbitrarily high codimension; and

(ii) the map $\pi^{*}: A_{*} Y \rightarrow A_{*} B$ is an isomorphism.

Proof. Since $G$ acts linearly on the quasiprojective scheme $X$, the quotient stack $Y$ has the property that every coherent sheaf admits a surjective map from a locally free sheaf. So, by [D, Proposition 1.4.15], $B$ is isomorphic to a globally presented vector bundle stack $[E / F]$ for some morphism $F \rightarrow E$ of vector bundles on $Y$. To establish (i) and (ii), it suffices to consider the case when $E$ is zero, i.e., $B=B F$. Now we consider the vector bundle $R \rightarrow B$ given by the bundle $F \oplus 1$ on $Y$ with $F$-action given by $f:(x, t) \mapsto(x+t f, t)$. There is a projection map $r: R \rightarrow \mathbb{A}^{1}$, and the fiber $r^{-1}\left(\mathbb{A}^{1} \backslash\{0\}\right)$ is isomorphic to $Y \times\left(\mathbb{A}^{1} \backslash\{0\}\right)$. Hence the composite map $R^{\oplus n} \rightarrow B \rightarrow Y$, restricted to $r^{\times n}\left(\mathbb{A}^{n} \backslash\{0\}\right)$, is representable, and so for a suitable vector bundle $E \rightarrow B$ (we take $E$ to be the pullback from $B G$ of a suitable representation bundle of $G$, as in [E-G1]), the total space of $R^{\oplus n} \oplus E$ possesses an open substack that is representable by a scheme and has complement of codimension $\geq n$. This establishes (i). Statement (ii) is a consequence of Corollary 3.5.3, since the structure map $\varphi: Y \rightarrow B$ is an affine bundle and we have $\pi \circ \varphi=1_{Y}$.

\section{Extended excision axiom}

\subsection{A first higher Chow theory}

We need some sort of a first higher Chow group in order to be able to extend the excision sequence one place to the left. We take as motivation the long exact localization sequence coming from the Gersten complex of schemes.

Notation 5.1.1. In this section, $A_{j}(X ; 1)$ denotes, for a scheme $X$, the kernel of $\partial: W_{j} X \rightarrow Z_{j} X$, modulo the subgroup generated by tame symbols of elements of $K_{2}(k(Y))$ for all $(j+2)$-dimensional integral closed subschemes $Y$ of $X$.

Remark 5.1.2. On a separated scheme $X$, we recognize $A_{j}(X ; 1)$ as an $E^{2}$ term of the Quillen spectral sequence of $K$-theory. Formal properties, cf. [Gi2], imply that if $X$ is a scheme (separated or not), and if $\pi: E \rightarrow X$ is a flat morphism whose pointwise fibers are $r$-dimensional affine spaces, then the induced map $A_{j}(X ; 1) \rightarrow A_{j+r}(E ; 1)$ is an isomorphism.

The failure of descent for $K$-theory in the smooth topology means we cannot apply the machinery of the Quillen spectral sequence directly to stacks. We must resort to a 
cycle-based complex, which we can show to be quasi-isomorphic (in the needed range) to the Gersten complex on a scheme base.

The starting point for cycle-based complexes is the theory of Bloch's higher Chow groups [Bl1, Bl2]. Let us recall that the $n^{\text {th }}$ term in the Bloch complex for a scheme $X$ is the free abelian group of cycles on $X \times \triangle^{n}$ meeting boundary cycles properly; $\triangle^{n}$ denotes the algebraic $n$-simplex $\left(\simeq \mathbb{A}^{n}\right)$ and the boundary cycles are copies of $X \times \triangle^{m}$ for $m<n$. In particular, when $n=1$ the boundary consists of just two zero-simplices (points), and the condition of proper intersection says nothing other than that no component of a cycle be contained in either of the boundary components. Thus the rightmost terms in the Bloch complex are

$$
\cdots \rightarrow Z_{j+1} X \times\left(\mathbb{A}^{1} \backslash\{2 \text { points }\}\right) \rightarrow \mathrm{Z}_{\mathrm{j}} \mathrm{X} \rightarrow 0
$$

where the final boundary map is the difference of two cycle-level specialization maps.

We identify $\mathbb{A}^{1} \backslash\{2$ points $\}$ with $R:=\mathbb{P}^{1} \backslash\{0,-1, \infty\}$, and we denote by $\pi$ the projection $X \times R \rightarrow X$. For $t \in \mathbb{P}^{1}$, we define $\partial_{t}: Z_{j+1} X \times \mathbb{P}^{1} \rightarrow Z_{j} X$ to be the cycle-level pullback via $X \simeq X \times\{t\} \rightarrow X \times \mathbb{P}^{1}$. Since $\partial_{t}$ kills any cycle supported in a fiber of $\pi$, there is an induced map $\partial_{t}: Z_{j+1} X \times R \rightarrow Z_{j} X$. We set $\partial=\partial_{0}-\partial_{\infty}$. Then $\partial: Z_{*} X \times R \rightarrow Z_{*} X$ is the rightmost map in (5.1.1).

We remark that there is a variant of (5.1.1), obtained by moding out by degenerate cycles (so, e.g., in term 1 we mod out by $\pi^{*} Z_{j} X$ ). The so-called normalized complex which results is quasi-isomorphic to the original complex.

Let $X$ be a stack. We introduce a cycle complex which has the same groups in positions 0 and 1 as the normalized Bloch complex, and we put the group $Z_{*}\left(X \times T^{2}\right)$ in position 2 to yield

$$
Z_{j+2}\left(X \times T^{2}\right) \stackrel{\partial}{\rightarrow} Z_{j+1}(X \times R) / \pi^{*} Z_{j} X \stackrel{\partial}{\rightarrow} Z_{j} X \rightarrow 0 .
$$

Here $T^{2}$ denotes the two-dimensional torus $\left(\mathbb{A}^{1} \backslash\{0\}\right)^{2}$. The complex will not go beyond position 2 .

The map $\partial: Z_{j+1}(X \times R) / \pi^{*} Z_{j} X \rightarrow Z_{j} X$ is the map $\partial_{0}-\partial_{\infty}$ of the Bloch complex. We give a definition of the boundary map $\partial: Z_{j+2}\left(X \times T^{2}\right) \rightarrow Z_{j+1}(X \times R) / \pi^{j} Z_{*} X$, so that (5.1.2) is a complex. We start by fixing an orientation convention on toric compactifications of $T^{2}$. Let $Y$ be a nonsingular two-dimensional complete toric variety, with corresponding fan $\Delta$. Let $(u, v) \in N=\mathbb{Z}^{2}$ be a generator of a ray $\rho \in \Delta$. Let $\left(u^{\prime}, v^{\prime}\right)$ be the generator of the ray immediately preceding $\rho$ via the counterclockwise ordering of rays, and let $\left(u^{\prime \prime}, v^{\prime \prime}\right)$ generate the ray immediately following $\rho$. If $x$ and $y$ denote coordinates on $T^{2} \subset Y$, then the maximal cone of $\Delta$ preceding $\rho$ corresponds to the toric affine chart Spec $k\left[x^{v} y^{-u}, x^{-v^{\prime}} y^{u^{\prime}}\right]$, and the maximal cone of $\Delta$ following $\rho$ corresponds to the toric affine chart Spec $k\left[x^{v^{\prime \prime}} y^{-u^{\prime \prime}}, x^{-v} y^{u}\right]$. Corresponding to $\rho$ is a toric divisor $D_{\rho}$ of $Y$. We define our orientation convention to be the identification of $D_{\rho}$ with $\mathbb{P}^{1}$ via Spec $k\left[x^{v} y^{-u}\right] \rightarrow \mathbb{A}^{1}=\mathbb{P}^{1} \backslash\{\infty\}$ and Spec $k\left[x^{-v} y^{u}\right] \rightarrow \mathbb{P}^{1} \backslash\{0\}$. There are two possible orientation conventions, but the point of $D_{\rho}$ corresponding to $\{-1\}$ 
in $\mathbb{P}^{1}$ is independent of this choice. Thus there is a natural subset $D_{\rho}^{\circ} \subset D_{\rho}$, defined as the complement in $D_{\rho}$ of the torus-fixed points and the point corresponding to $\{-1\}$. Our convention specifies an isomorphism $R \rightarrow D_{\rho}^{\circ}$.

Suppose $V$ is an integral closed substack of $X \times \mathbb{P}^{2}$ such that $V$ meets $X \times T^{2}$ nontrivially, By induction on excess of intersection (cf. [F, §2.4]), we find that there is a finite sequence of blowups at torus-fixed points $Y \rightarrow \mathbb{P}^{2}$ such that the proper transform $\widetilde{V}$ of $V$ meets the toric divisors of $X \times Y$ properly. For each $\rho$ in the fan defining $Y$ we can pull back $[\widetilde{V}]$ via the composite

$$
X \times R \stackrel{\sim}{\rightarrow} X \times D_{\rho}^{\circ} \rightarrow X \times D_{\rho} \rightarrow X \times Y
$$

to obtain a cycle $\partial_{\rho}([V]) \in Z_{*} X \times R$. By dimension reasoning, the sum $\sum_{\rho} \partial_{\rho}([V])$ in $Z_{*}(X \times R) / \pi^{*} Z_{*} X$ is independent of the choice of $Y$. Because of proper intersection, we have $\partial\left(\sum_{\rho} \partial_{\rho}([V])\right)=0$. Hence, if we define $\partial=\sum_{\rho} \partial_{\rho}$, then (5.1.2) is a complex.

Definition 5.1.3. Let $X$ be a stack. We denote by $\underline{A}_{j}^{\circ} X$ the homology group in the first position of the complex (5.1.2).

Remark 5.1.4. The boundary maps clearly respect proper pushforward and flat pullback, making the association $X \mapsto \underline{A}_{*}^{\circ} X$ functorial for proper pushforward and flat pullback.

Let us examine the case $X=\operatorname{Spec} k$. Let $C \subset T^{2}$ be an integral subscheme of dimension 1. Then $C$ is given as the zero locus of a single function $f \in k\left[x^{ \pm 1}, y^{ \pm 1}\right]$. It is easy to describe a toric variety $Y$ suitable for computing $\partial([C])$. We let $N=\mathbb{Z}^{2}$, and $M=\operatorname{Hom}(N, \mathbb{Z})$. If $f=\sum a_{\mu \nu} x^{\mu} y^{\nu}$, we let $\Gamma$ be the Newton polygon of $f$, that is, the convex hull in $M \otimes \mathbb{R}$ of the set of points $(\mu, \nu)$ for which $a_{\mu \nu} \neq 0$. There exists a finite collection of half-planes $\left\{H_{\rho_{i}}^{+}\right\}$which cut out $\Gamma$, where for $\rho \in N$ we define

$$
\begin{aligned}
\lambda_{\rho} & =\min \{\langle\zeta, \rho\rangle \mid \zeta \in \Gamma\}, \\
H_{\rho} & =\left\{\zeta \in M \otimes \mathbb{R} \mid\langle\zeta, \rho\rangle=\lambda_{\rho}\right\}, \\
H_{\rho}^{+} & =\left\{\zeta \in M \otimes \mathbb{R} \mid\langle\zeta, \rho\rangle \geq \lambda_{\rho}\right\},
\end{aligned}
$$

where $\langle$,$\rangle denotes the pairing of M$ and $N$. If $p$ and $q$ lie in the set $\operatorname{Vert}(\Gamma)$ of vertices of $\Gamma$, let us say that $q$ follows $p$ if the line segment joining $p$ and $q$ is an edge of $\Gamma$, and if for all $r \in \operatorname{int}(\Gamma)$, the signed angle from ray $p q$ to ray $p r$ is positive.

Proposition 5.1.5. Suppose $\Gamma=\bigcap_{i} H_{\rho_{i}}^{+}$. If $\Delta$ is a complete nonsingular fan in $N$ which contains all the rays generated by the $\rho_{i}$, then the closure $\widetilde{C}$ of $C$ in the toric variety corresponding to $\Delta$ meets the toric divisors properly. Moreover, if $N: Z_{0} R \rightarrow$ $k^{*}$ is defined by sending a zero-cycle $[Z]$, with $Z \subset R$ integral, to the image under the norm map $k(Z)^{*} \rightarrow k^{*}$ of the function $(-t)$, where $t$ is the restriction to $R$ of the natural coordinate on $\mathbb{A}^{1}$, then we have

$$
N\left(\partial_{\rho}([C])\right)= \begin{cases}a_{p} / a_{q} & \text { if } H_{\rho} \cap \operatorname{Vert}(\Gamma)=\{p, q\} \text { such that } q \text { follows } p, \\ 1 & \text { otherwise. }\end{cases}
$$


Proof. Proper intersection is equivalent to saying that $\widetilde{C}$ does not contain any of the torus fixpoints. Let $\rho_{1}, \ldots, \rho_{m}$ be generators of the rays of $\Delta$, arranged in counterclockwise order. The hypotheses guarantee that $H_{\rho_{i}}$ and $H_{\rho_{i+1}}$ intersect at a vertex of $\Gamma$, for each $i$. Thus the equation for $\tilde{C}$ in a neighborhood of the $i^{\text {th }}$ torus fixpoint has a nontrivial constant term. Moreover, $\widetilde{C} \cap D_{\rho_{i}} \neq \emptyset$ if and only if $H_{\rho_{i}}$ contains two vertices of $\Gamma$, say $p=H_{\rho_{i-1}} \cap H_{\rho_{i}}$ and $q=H_{\rho_{i}} \cap H_{\rho_{i+1}}$. For such $i$, the equation defining the scheme $C \cap D_{i}$ is a polynomial with leading term $a_{q}$ and constant term $a_{p}$, and hence the formula.

Corollary 5.1.6. The composite $Z_{1} T^{2} \rightarrow Z_{0} R \rightarrow k^{*}$ is the zero map, and the induced map $Z_{0} R / \partial\left(Z_{1} T^{2}\right) \rightarrow k^{*}$ is an isomorphism.

Proof. The first claim is an immediate consequence of Proposition 5.1.5. For the second claim, the map is clearly surjective, so we need only verify that a general zero-cycle on $R$ is equal, modulo $\partial$ of an element of $Z_{1} T^{2}$, to a cycle of the form $[\{r\}]$ for some $r \in k^{*}$. A general effective zero-cycle is of the form $[Z]$ where $Z$ is the zero locus of some polynomial $t^{n}+a_{1} t^{n-1}+\cdots+a_{n}$. If $C$ is the zero locus of $x^{n}+a_{1} x^{n-1}+\cdots+a_{n}+y$, then $\partial([C])=[Z]+\left[\left\{r^{-1}\right\}\right]$, where $r=-a_{n}$. In particular, as well, $[\{r\}]+\left[\left\{r^{-1}\right\}\right]=0$. Thus the class of a general zero-cycle has a representative of the form $[\{r\}]$ for some $r \in k^{*}$.

Proposition 5.1.7. There is a natural isomorphism of functors on schemes $\underline{A}_{*}^{\circ}(-) \rightarrow$ $A_{*}(-; 1)$.

Proof. Let $X$ be a scheme, and let $j$ be an integer. We propose maps $N$ yielding a morphism of complexes

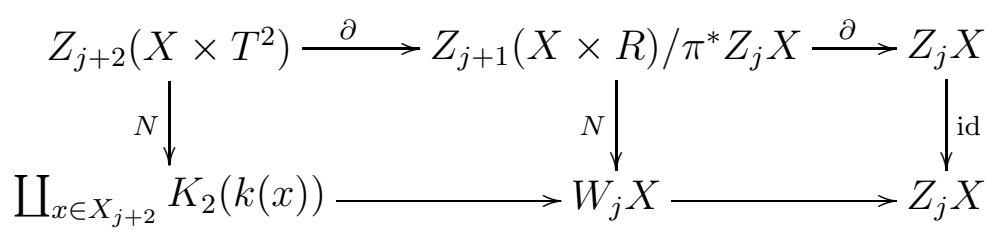

from the cycle complex (5.1.2) to the Gersten complex of $X$ (we denote by $X_{j}$ the set of points $x \in X$ having $\operatorname{dim} \overline{\{x\}}=j$ ). The middle vertical map is the norm map, preceded by the involution $t \mapsto-t$ on $R$ (as in Proposition 5.1.5). For the map on the left, let $V$ be an integral closed subscheme of $X \times \mathbb{P}^{2}$ of dimension $(j+2)$, and consider the image $p(V)$ under projection $p: X \times \mathbb{P}^{2} \rightarrow X$. There are 3 cases:

(i) $\operatorname{dim} p(V)=j$, i.e., $V=U \times \mathbb{P}^{2}$ for some $U \subset X$, and clearly $[V]$ is killed by $\partial$; we define $N([V])=0$.

(ii) $\operatorname{dim} p(V)=j+1$, so $N \circ \partial([V])$ consists just of a rational function on $p(V)$. To compute, we may replace $X$ by the generic point of $p(V)$, and by Corollary 5.1.6, $N(\partial([V]))=0$; we define $N([V])=0$. 
(iii) $\operatorname{dim} p(V)=j+2$, so $k(p(V)) \rightarrow k(V)$ is a finite field extension, and there is a norm map $K_{2}(k(V)) \rightarrow K_{2}(k(p(V)))$. We send $[V]$ to the image under the norm map of the symbol $\{-x,-y\} \in K_{2}(k(V))$, where $x$ and $y$ denote the coordinate functions on $T^{2}$.

We have defined a map $N: Z_{j+2}\left(X \times \mathbb{P}^{2}\right) \rightarrow \coprod_{x \in X_{j+2}} K_{2}(k(x))$. Since this map vanishes on cycles supported in the complement of $X \times T^{2}$, there is an induced map $N$ on $Z_{j+2}\left(X \times T^{2}\right)$, as indicated in (5.1.4). Now, commutativity of (5.1.4) follows from the fact that the norm map of $K$-theory commutes with the tame symbol.

We claim, now, that $N$ induces isomorphisms on the zeroth and first homology groups. The map on zeroth homology groups is clearly the identity map on $A_{*}^{\circ} X$. Let us consider the induced map $N_{1}$ on first homology groups. The vertical maps $N$ in (5.1.4) are surjective (given any rational function $r$ on an integral closed subscheme of $X$, the graph of $(-r)$ specifies an element of $Z_{*}(X \times R)$ whose image under $N$ is the specified rational function; a similar argument with pairs of functions applies for the map on the left), so $N_{1}$ is surjective, and to show $N_{1}$ is injective it suffices to show that if $\alpha \in Z_{*}(X \times R) / \pi^{*} Z_{*} X$ satisfies $N(\alpha)=0$ in $W_{*} X$, then $\alpha$ lies in the image of $\partial$. For this we are easily reduced to the case $X=\operatorname{Spec} k$, and by Corollary 5.1.6, $N_{1}: \underline{A}_{*}^{\circ}(\operatorname{Spec} k) \rightarrow A_{*}(\operatorname{Spec} k ; 1)$ is an isomorphism.

We wish to repeat the construction of section 2.1, starting with the functor $\underline{A}_{*}^{\circ}$ rather than $A_{*}^{\circ}$. Thus we need to study what happens when we have two surjections of vector bundles $E \rightarrow F$ on a stack $X$.

Lemma 5.1.8. Let $S=T^{2} \backslash\{(x, y) \mid x+y+1=0\}$. The map $\partial: Z_{*} X \times T^{2} \rightarrow$ $Z_{*}(X \times R) / \pi^{*} Z_{*} X$ factors through $Z_{*}(X \times S)$. The map $\partial$ also factors through $Z_{*}(X \times R \times R)$.

Proof. Suppose $\alpha \in Z_{*} X \times T^{2}$ has support in the locus specified by $x+y+1=0$. Then the closure of $\alpha$ in $Z_{*} X \times \mathbb{P}^{2}$ meets the boundary divisors properly, and the pullback of $\alpha$ under (5.1.3) is zero for each of the 3 boundary cycles of $\mathbb{P}^{2}$. A similar argument applies if $\alpha$ has support in the locus given by $(x+1)(y+1)=0$, using $\mathbb{P}^{1} \times \mathbb{P}^{1}$ in place of $\mathbb{P}^{2}$.

There is a map $S \rightarrow R$, given by $(x, y) \mapsto x+y$. If $\sigma$ denotes the induced map $X \times S \rightarrow X \times R$, then for any $\alpha \in Z_{*}(X \times R), \sigma^{*} \alpha$ satisfies $\partial\left(\sigma^{*} \alpha\right)=\alpha+\tau^{*} \alpha$, where $\tau$ is the map $X \times R \rightarrow X \times R$ induced by the involution $t \mapsto t^{-1}$ on $R$.

Proposition 5.1.9. Let $X$ be a stack, let $E$ and $F$ be vector bundles on $X$, and let $\varphi$ and $\psi$ be surjections of vector bundles from $E$ to $F$. Then the maps $\underline{A}_{*}^{\circ} F \rightarrow \underline{A}_{*}^{\circ} E$ induced by $\varphi$ and $\psi$ are the same.

Proof. Let $r$ and $s$ denote free parameters; then $r \varphi+s \psi$ is a vector bundle surjection from $E \times \mathbb{A}^{2}$ to $F$. Consider the restriction of $r \varphi+s \psi$ to $E \times\left(\mathbb{A}^{2} \backslash P\right)$, where 
$P=\left\{(r, s) \in A^{2} \mid r+s=0\right\}$. If we let $Q=\left\{(r, s) \in \mathbb{A}^{2} \mid r+s=1\right\}$, then there is a smooth surjective map $E \times\left(\mathbb{A}^{2} \backslash P\right) \rightarrow E \times Q$ such that

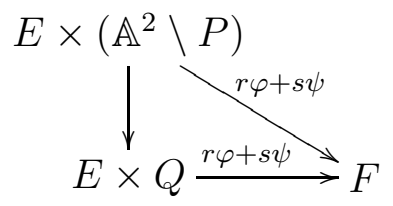

commutes, and hence the restriction of $r \varphi+s \psi$ to $E \times Q$ is smooth. Now let $\lambda: R \rightarrow Q$ be the map $t \mapsto t /(t+1)$. There are induced maps

$$
Z_{*}(F \times R) \stackrel{\left(\left.(r \varphi+s \psi)\right|_{Q}\right)^{*}}{\longrightarrow} Z_{*}(E \times R \times Q) \stackrel{\lambda^{*}}{\longrightarrow} Z_{*}(E \times R \times R)
$$

and now by a direct computation we have

$$
\partial\left(\lambda^{*}\left(\left.(r \varphi+s \psi)\right|_{Q}\right)^{*} \alpha+\sigma^{*} \varphi^{*} \alpha\right)=\psi^{*} \alpha-\varphi^{*} \alpha
$$

for any $\alpha \in Z_{*}(F \times R) / \pi^{*} Z_{*} F$.

Corollary 5.1.10. There is a functor $X \mapsto \underline{A}_{*} X$ and a natural transformation of functors $\underline{A}_{*}^{\circ} \rightarrow \underline{A}_{*}$. The natural map $\underline{A}_{*}^{\circ} X \rightarrow \underline{A}_{*} X$ is an isomorphism when $X$ is a scheme.

Proof. Proposition 5.1.9 implies that for a stack $Y,\left\{\underline{A}_{j+\mathrm{rk} E}^{\circ} E\right\}$ forms a direct system over the directed set $\mathfrak{B}_{Y}$ of Definition 2.1.3. There is, therefore, a functor $Y \mapsto$ $\underline{\widehat{A}}_{k} Y:=\underline{\lim }_{\mathfrak{B}_{Y}} \underline{A}_{j+\mathrm{rk} E}^{\circ} E$ and a natural map $\underline{A}_{j}^{\circ} Y \rightarrow \underline{\hat{A}}_{j} Y$. For projective morphism $f: X \rightarrow Y$, we define the groups $\widehat{B}_{j} X$ as in Definition 2.1.9, and then we set

$$
\underline{A}_{j} Y=\underline{\lim }_{\mathfrak{A}_{Y}}\left(\underline{\widehat{A}}_{j} X / \underline{B}_{j} X\right) \text {. }
$$

As in Remark 2.1.16, we see that the natural map $\underline{A}_{*}^{\circ} Y \rightarrow \underline{A}_{*} Y$ is an isomorphism for any scheme $Y$.

\subsection{The connecting homomorphism}

Let $X$ be a stack, and let $Y$ be a closed substack of $X$ with complement $U$. From the exact sequence of complexes

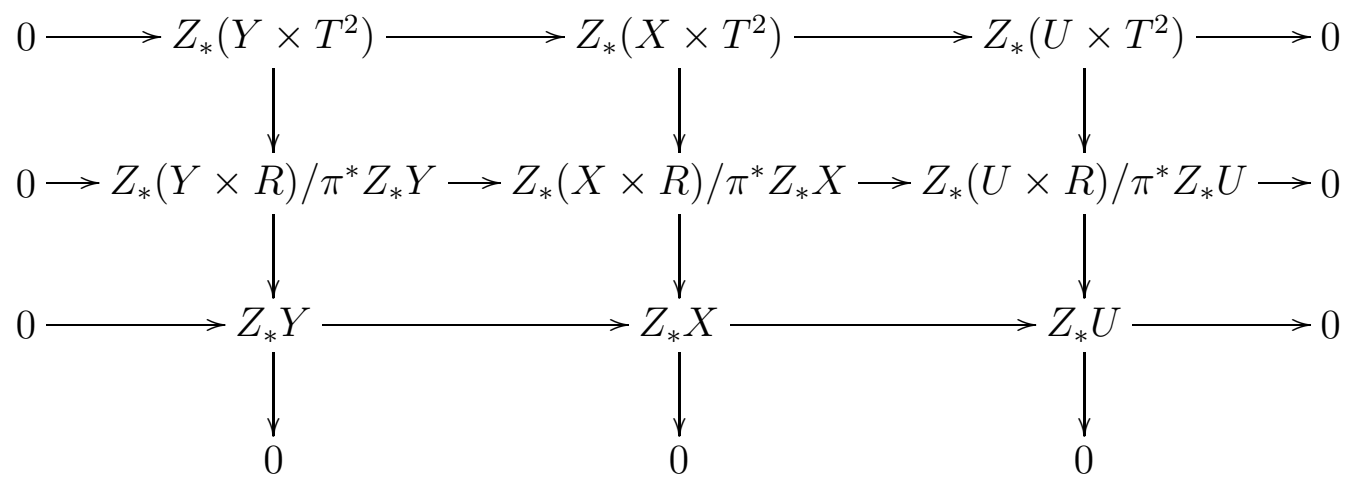


there is a long exact sequence of cohomology groups

$$
\underline{A}_{*}^{\circ} Y \rightarrow \underline{A}_{*}^{\circ} X \rightarrow \underline{A}_{*}^{\circ} U \stackrel{\delta}{\rightarrow} A_{*}^{\circ} Y \rightarrow A_{*}^{\circ} X \rightarrow A_{*}^{\circ} U \rightarrow 0 .
$$

A bit of checking shows that the connecting homomorphism $\delta$ induces a map $\delta$ in the complex

$$
\underline{A}_{*} U \stackrel{\delta}{\rightarrow} A_{*} Y \rightarrow A_{*} X \rightarrow A_{*} U \rightarrow 0
$$

The map $\delta$ commutes with flat pullback and projective pushforward.

Proposition 5.2.1. Assume $U$ is a global quotient stack. Then the complex (5.2.2) is exact.

Proof. Suppose $h: V \rightarrow Y$ is projective, $\alpha \in \hat{A}_{*} V$, and $i_{*}(h, \alpha)=0$ in $A_{*} X$, where $i: Y \rightarrow X$ denotes inclusion. After adding components to $V$, there must exist a projective morphism $f: T \rightarrow X$ and projective morphisms $p_{1}, p_{2}: S \rightarrow T$ such that $f \circ p_{1}$ is 2-isomorphic to $f \circ p_{2}$, such that $j: V \rightarrow Y$ identifies $V$ with $Y \times_{X} T$, and such that there exist $\beta_{1} \in \hat{A}_{*}^{p_{1}} S$ and $\beta_{2} \in \hat{A}_{*}^{p_{2}} S$ such that $\iota_{p_{1}}\left(\beta_{1}\right)=\iota_{p_{2}}\left(\beta_{2}\right)$ in $\hat{A}_{*} S$ and

$$
p_{2 *} \beta_{2}-p_{1 *} \beta_{1}=j_{*} \alpha .
$$

Suppose $U \simeq[W / G]$. Fix a representation bundle $B$ on $B G$. There exists a projective modification $\pi: \widetilde{X} \rightarrow X$ such that some vector bundle $E$ on $\tilde{X}$ restricts to the pullback of $B$ to $U$. Suppose we are in the special case where $f$ factors as $\pi \circ \tilde{f}$, and $\tilde{f} \circ p_{1}$ is 2 -isomorphic to $\tilde{f} \circ p_{2}$. Then there exists $\beta \in A_{*}^{\circ}\left(\tilde{f}^{*} E\right)^{\oplus n}$ for suitable $n$ such that, with the fiber diagram

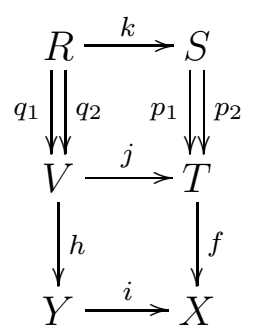

there are $\gamma_{i} \in \hat{A}_{*}^{j \circ q_{i}} R$ such that $\beta_{i}=\beta+k_{*} \gamma_{i}$ in $\hat{A}_{*}^{p_{i}} S$ for $i=1,2$. Then, in $A_{*} X$, we have

$$
(h, \alpha)=\left(h, \alpha+q_{1 *} \gamma_{1}-q_{2 *} \gamma_{2}\right)+\left(h \circ q_{1}, \gamma_{2}-\gamma_{1}\right),
$$

and each term on the right individually lies in the image of $\delta$.

We now deduce the general case from the special case. Suppose (5.2.3) holds, with $f: T \rightarrow X$ a general projective morphism. There exists a projective modification 
$\sigma: \widetilde{T} \rightarrow T$ such that $f \circ \sigma$ factors through $\widetilde{X}$. We can make a modification $\tau: \widetilde{S} \rightarrow S$ so that

$$
(\widetilde{S} \rightrightarrows \widetilde{T} \rightarrow \widetilde{X}) \rightarrow(S \rightrightarrows T \rightarrow X)
$$

is a morphism of coequalizer diagrams. There exists $\tilde{\beta}_{i} \in \widehat{A}_{*}^{p_{i} \circ \tau} \widetilde{S}$ such that $\tau_{*} \tilde{\beta}_{i}=$ $\beta_{i}+k_{*} \gamma_{i}$ holds in $\widehat{A}_{*}^{p_{i}} S$, for some $\gamma_{i} \in \widehat{A}^{p_{i} \circ k} R$. If $\tilde{k}$ denotes the pullback of $k$, then we have $\iota\left(\tilde{\beta}_{2}\right)-\iota\left(\tilde{\beta}_{1}\right)=\tilde{k}_{*} \varepsilon$ for some $\varepsilon \in \widehat{A}_{*}^{\tau} \tilde{\tilde{k}} \widetilde{R}$.

Let $\tilde{\tau}: \widetilde{R} \rightarrow R$ denote the pullback of $\tau$. By the special case above, we have, in $A_{*} Y / \delta\left(\underline{A}_{*} U\right)$,

$$
\begin{aligned}
0 & =\left(h, \alpha+q_{2 *} \gamma_{2}-q_{1 *} \gamma_{1}\right)-\left(h \circ q_{1} \circ \tilde{\tau}, \varepsilon\right) \\
& =(h, \alpha)+\left(h \circ q_{1}, \gamma_{2}-\gamma_{1}-\tilde{\tau}_{*} \varepsilon\right) \\
& =(h, \alpha) . \quad \square
\end{aligned}
$$

\subsection{Homotopy invariance for vector bundle stacks}

Proposition 5.3.1. Let $X$ be a quasiprojective scheme, let $G$ be a smooth connected linear algebraic group acting linearly on $X$, let $Y=[X / G]$, and let $\pi: B \rightarrow Y$ be a vector bundle stack. Then $\pi^{*}: \underline{A}_{*} Y \rightarrow \underline{A}_{*} B$ is an isomorphism.

Proof. Routine, using scheme approximations (Proposition 4.5 .8 (i)), plus Proposition 5.1.7 and homotopy invariance of $A_{*}(-; 1)$.

With the machinery developed so far, we finally arrive at a proof of the homotopy property for vector bundle stacks on stacks which admit stratifications by global quotients.

Proposition 5.3.2. Let $X$ be a stack which admits a stratification by global quotients, and let $\pi: E \rightarrow X$ be a vector bundle stack. Then $\pi^{*}: A_{*} X \rightarrow A_{*} E$ is an isomorphism.

Proof. We proceed by noetherian induction. Let $U$ be a nonempty open substack of $X$ such that $U$ is isomorphic to the quotient of a quasiprojective scheme by the linearized action of a smooth connected linear algebraic group.

Let $B$ denote the restriction of $E$ to $U$. By Proposition 4.5.8 (i), $B$ is a global quotient. Let $Y=X \backslash U$, and let $F$ denote the restriction of $E$ to $Y$. Pullback induces a morphism of complexes

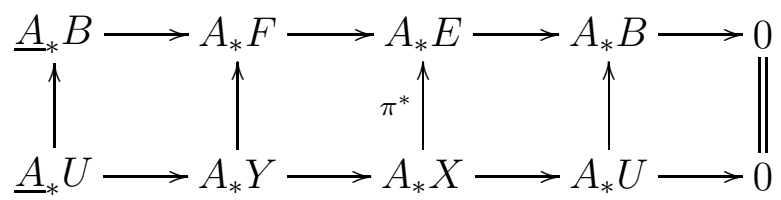

and by Proposition 5.2.1 both the bottom complex and the top complex are exact. From Propositions 4.5.8 (ii) and 5.3.1 and the induction hypothesis, the five lemma implies that $\pi^{*}: A_{*} X \rightarrow A_{*} E$ is an isomorphism. 


\section{Intersection theory}

\subsection{Intersections on Artin stacks}

The construction of the deformation space to a regular local immersion (section 4.1) can be generalized to an arbitrary morphism that is representable and locally separated. Suppose $f: F \rightarrow G$ is representable and locally separated. Then there is a deformation space $M_{F}^{\circ} G \rightarrow \mathbb{P}^{1}$ with general fiber $G$ and special fiber the normal cone stack to $f$. We will apply this construction to the diagonal of an Artin stack to obtain $M_{F}^{\circ}(F \times F) \rightarrow \mathbb{P}^{1}$. When $F$ is smooth, the special fiber is the tangent bundle stack $T F$, and this construction leads to an intersection product on $F$ provided that $F$ admits a stratification by global quotient stacks.

Here is the construction. Let $f: F \rightarrow G$ be a representable, locally separated morphism. It is not hard to see that there exists a commutative square

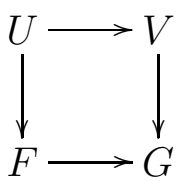

such that $U$ and $V$ are schemes and such that the top arrow is a closed immersion.

By the hypotheses on $f$, the induced map $R:=U \times_{F} U \rightarrow U \times_{G} V$ is a locally closed immersion, and is in fact a regular immersion. We let $S=V \times_{G} V$, with projections $q_{1}$ and $q_{2}$ to $V$. The composite $R \rightarrow U \times_{G} V \rightarrow S$ is also a locally closed immersion, so there is a deformation space $M_{R}^{\circ}$, flat over $\mathbb{P}^{1}$, with a map $M_{R}^{\circ} \rightarrow S$ that is representable and separated.

By the universal property of blowing up, the morphism $M_{R}^{\circ} \rightarrow V \times \mathbb{P}^{1}$ induced by $q_{i}$ factors through $\mathrm{B} \ell_{U} V \times \mathbb{P}^{1}$, and in fact, factors through $M_{U}^{\circ} V=\mathrm{B} \ell_{U} V \times \mathbb{P}^{1} \backslash \mathrm{B} \ell_{U} V$. So, we get morphisms $r_{i}: M_{R}^{\circ} S \rightarrow M_{U}^{\circ} V$ over $\mathbb{P}^{1}$. for $i=1,2$. The restriction of $r_{i}$ over $\mathbb{P}^{1} \backslash\{0\}$ is clearly smooth, and $r_{i} \times_{\mathbb{P}^{1}}\{0\}$ factors as

$$
C_{R} S \rightarrow\left(C_{U \times_{V} S} S\right) \times_{U \times_{V} S} R \simeq C_{U} V \times_{U} R \rightarrow C_{U} V
$$

and hence is also smooth (the first map appears in the exact sequence of cones $0 \rightarrow$ $\left.\left.N_{R}\left(U \times_{V} S\right) \rightarrow C_{R} S \rightarrow C_{U \times_{V} S} S\right|_{R} \rightarrow 0\right)$. The maps $r_{i}$ are flat by the local criterion for flatness, and hence are smooth. The maps $r_{i}$ together with additional maps obtained in the expected fashion determine a groupoid $\left[M_{R}^{\circ} S \rightrightarrows M_{U}^{\circ} V\right]$ which specifies a stack that we denote $M_{F}^{\circ} G$.

Now, suppose $f: F \rightarrow G$ is a representable, locally separated morphism such that the normal cone stack to $f$ is a vector bundle stack of constant (virtual) rank $d$, and suppose $G^{\prime} \rightarrow G$ is a morphism such that $F^{\prime}:=F \times_{G} G^{\prime}$ admits a stratification by global quotient stacks (this is the case, for instance, if $G^{\prime}$ admits a stratification by global quotient stacks). Then, by the construction of section 4.1 combined with the homotopy property for vector bundle stacks over a stack which admits a 
stratification by global quotient stacks, we obtain the refined Gysin homomorphism $A_{*} G^{\prime} \rightarrow A_{*-d} F^{\prime}$.

The proofs of basic properties about Gysin homomorphisms (compatibility with flat pullback and projective pushforward, commutativity, and functoriality) apply unchanged to let us deduce these properties for the refined Gysin homorphisms appearing in this section.

Let $F$ be a stack which admits a stratification by global quotient stacks. We remark on several instances when the Gysin homomorphism to a representable locally separated morphism $f: F \rightarrow G$ agrees with the map $f^{*}$ constructed by other methods. First, when $f$ is smooth, or more generally flat and l.c.i., the Gysin homomorphism is the same as flat pullback: the virtual normal bundle is a vector bundle stack with surjective zero section $\varphi: \mathfrak{N} \rightarrow F$ and the Fulton-MacPherson produces, starting with the cycle $[Z]$ for some $Z \subset G$, the cycle $\left[\varphi^{-1}\left(f^{-1}(Z)\right)\right]$ in $\mathfrak{N}$. Next, when $f$ is a regular local immersion, the virtual normal bundle is just the usual normal bundle, so the construction reduces to the usual Fulton-MacPherson construction. Finally, let $f$ be a l.c.i. morphism which admits a global factorization as a regular immersion $i: F \rightarrow P$ followed by a representable locally separated smooth morphism $g: P \rightarrow G$. Then there is a morphism of vector bundles $i^{*} T_{P / G} \rightarrow N_{F} P$, and the normal bundle stack to $f$ admits the global presentation $\left[N_{F} P / i^{*} T_{P / G}\right]$. Now functoriality of the Gysin homomorphism gives us a new way to see that the definition of Gysin map to a l.c.i. morphism given in $[\mathrm{F}], f^{*}=i^{*} \circ g^{*}$, is independent of the choice of factorization. For an l.c.i. morphism of schemes which does not admit a global factorization, the Gysin map has previously been constructed in [Gi3] using higher $K$-theory.

The diagonal morphism of a smooth Artin stack is representable, separated, and l.c.i. When $F$ is a smooth Artin stack which admits a stratification by global quotient stacks, the Gysin homomorphism to the diagonal $F \rightarrow F \times F$ induces a ring structure on $A_{*} F$. This is the intersection product.

We can provide an answer to Conjecture 6.6 of [V2].

Theorem 6.1.1. Let $F$ be a smooth Artin stack which has quasi-finite diagonal, and let $M$ be a coarse moduli space for $F$. Then $M$ satisfies Alexander duality.

Proof. This is an immediate consequence of the refined Gysin homomorphism to the diagonal $F \rightarrow F \times F$. In positive characteristic, de Jong's modifications play the role of resolution of singularity in showing that $\mathbb{Q}$-valued intersection operations commute.

Remark 6.1.2. The notion of Alexander duality in intersection theory was introduced in [V1]. For a scheme $X$ to satisfy Alexander duality means that $X$ behaves like a smooth scheme, as far as intersection theory with rational coefficients is concerned. In [V2] the characteristic zero case of Theorem 6.1.1 is deduced from the intersection theory on Deligne-Mumford stacks. 


\subsection{Virtual fundamental class}

Let $X$ be a Deligne-Mumford stack. The intrinsic normal cone $\mathfrak{C}_{X}$ is introduced in $[\mathrm{B}-\mathrm{F}]$ as a tool for constructing a virtual fundamental class in $A_{*}^{\circ} X \otimes \mathbb{Q}$ from a perfect obstruction theory. A perfect obstruction theory is an element of the derived category $E^{\bullet} \in D\left(\mathcal{O}_{X_{\text {ét }}}\right)$ of perfect amplitude contained in $[-1,0]$, together with a morphism $\varphi: E^{\bullet} \rightarrow L_{X}^{\bullet}$, where $L_{X}^{\bullet}$ denotes the cotangent complex on $X$, such that $h^{0}(\varphi)$ is an isomorphism and $h^{-1}(\varphi)$ is surjective. There is an associated geometric object $h^{1} / h^{0}\left(E^{\vee}\right)$, which is a vector bundle stack over $X$ : locally, we can write $E^{\bullet}$ as $\left[E^{-1} \rightarrow E^{0}\right]$, and the quotients $\left[E^{-1^{\vee}} / E^{0^{\vee}}\right]$ patch to form $h^{1} / h^{0}\left(E^{\vee}\right)$. The map $\varphi$ induces a closed immersion $\mathfrak{C}_{X} \rightarrow h^{1} / h^{0}\left(E^{\vee}\right)$.

The construction of the virtual fundamental class proceeds by starting with the cycle $\left[\mathfrak{C}_{X}\right]$ on $h^{1} / h^{0}\left(E^{\vee}\right)$ and "intersecting with the zero section" of $\pi: h^{1} / h^{0}\left(E^{\vee}\right) \rightarrow$ $X$ to obtain a cycle class on $X$. As remarked in $[\mathrm{B}-\mathrm{F}]$, to do this without intersection theory on stacks requires imposing the additional hypothesis that $E^{\bullet}$ admits a global presentation as $\left[E^{-1} \rightarrow E^{0}\right]$. Then we can pull back $\left[\mathfrak{C}_{X}\right]$ to the total space of $E_{1}:=E^{-1^{\vee}}$ and intersect with the zero section of $E_{1} \rightarrow X$.

The intersection theory of this preprint lets us remove this extra hypothesis.

Theorem 6.2.1. If $X$ is a Deligne-Mumford stack and $E^{\bullet}$ is a perfect obstruction theory on $X$ then there exists a unique element $\alpha \in A_{*}^{\circ} X \otimes \mathbb{Q}$ such that the pullback of $\alpha$ to $A_{*}\left(h^{1} / h^{0}\left(E^{\vee}\right)\right) \otimes \mathbb{Q}$ is equal to $\left[\mathfrak{C}_{X}\right]$.

Proof. This follows from Propositions 4.5.5 (iii) and 5.3.2.

Remark 6.2.2. Using only elementary techniques (sections 2 through 4 ), it is shown in $[\mathrm{Kr} 2]$ that when $X$ is a separated Deligne-Mumford stack, the map $\pi^{*}: A_{*} X \otimes \mathbb{Q} \rightarrow$ $A_{*} U \otimes \mathbb{Q}$ is an isomorphism. The proof uses the fact ([L-MB, (10.1)]) that there exists a finite surjective map from a scheme $T$ to $X$, so by applying Chow's lemma to $T$ we obtain a projective, generically finite, surjective map $f: Y \rightarrow X$ such that $Y$ is a quasiprojective scheme. The theorem is true for $Y$ (Proposition 4.5.8, with $G$ the trivial group) and for $Y \times_{X} Y$, so we deduce the result for $X$ by the analogue for $A_{*} \otimes \mathbb{Q}$ of the co-sheaf sequences in $A_{*}^{\circ} \otimes \mathbb{Q}$ of $[\mathrm{Ki}]$.

\subsection{Localization formula}

We describe a localization formula which is sufficient for computations in equivariant Chow groups over an algebraically closed base field of arbitrary characteristic. We follow [E-G2], which gives a localization formula for torus actions on schemes. Using the various functors introduced in this preprint, we can deduce a similar formula for torus actions on Deligne-Mumford stacks. In this section, all Chow groups are taken to have rational coefficients.

Lemma 6.3.1. Let $X$ be a stack with quasi-finite stabilizer. Then $A_{*}^{\circ} X \rightarrow A_{*} X$ is an isomorphism and $\underline{A}_{*}^{\circ} X \rightarrow \underline{A}_{*} X$ is surjective. 
Proof. It suffices to show that for any vector bundle $\pi: E \rightarrow X$ the pullback $A_{*}^{\circ} X \rightarrow$ $A_{*}^{\circ} E$ is an isomorphism and the map $\underline{A}_{*}^{\circ} X \rightarrow \underline{A}_{*}^{\circ} E$ is surjective. Suppose first that $X$ admits a finite flat cover by a scheme, $f: U \rightarrow X$. Then $f_{*} \circ f^{*}$ is an isomorphism, so we deduce that $\pi$ induces an isomorphism on $A^{\circ}$ and on $\underline{A}^{\circ}$ from the fact that this holds after pullback via $f$. By localization, noetherian induction, and the five lemma, we deduce the desired statements.

Corollary 6.3.2. Suppose $X$ has quasi-finite stabilizer. Then $A_{j} X=0$ for $j<0$ and $\underline{A}_{j} X=0$ for $j<-1$.

The localization property for a torus action on a Deligne-Mumford stack will be a consequence of exactness of the localization sequence (5.2.2), plus vanishing for dimension reasons. When the Deligne-Mumford stack is smooth and has finite stabilizer, we are able to prove that the fixed stack for the torus action is smooth with the aid of the next lemma, and the localization formula follows (the finite stabilizer hypothesis guarantees that the fixed locus is a closed substack).

Lemma 6.3.3. Let $A$ be a regular local $k$-algebra with residue field $A / \mathfrak{m} \simeq k$, let $\sigma$ be a $k$-algebra homomorphism $A \rightarrow A$, and suppose $\sigma^{n}=1_{A}$ for some positive integer $n$, where $n$ is prime to the characteristic of $k$ in case $k$ has positive characteristic. If $I_{\sigma}$ denotes the ideal generated by $f-\sigma f$ for all $f \in A$, then $I_{\sigma}$ is generated by part of a regular sequence of $A$.

Proof. Let $d=\operatorname{dim} A$. The result follows from two facts: (i) if $f_{1}, \ldots, f_{d}$ is a regular sequence, then $I_{\sigma}$ is generated by $\left(f_{1}-\sigma f_{1}\right), \ldots,\left(f_{d}-\sigma f_{d}\right)$; (ii) $f-\frac{1}{n}(f+$ $\left.\sigma f+\cdots+\sigma^{n-1} f\right) \in I_{\sigma}$ for any $f \in A$ (we have $f-\frac{1}{n}\left(f+\sigma f+\cdots+\sigma^{n-1} f\right)=$ $\left.(f-\sigma f)+\frac{n-1}{n}\left(\sigma f-\sigma^{2} f\right)+\cdots+\frac{1}{n}\left(\sigma^{n-1} f-f\right)\right)$. Using the idempotent projections $f \mapsto f-\frac{1}{n} \sum_{i=0}^{n-1} \sigma^{i} f$ and $f \mapsto \frac{1}{n} \sum_{i=0}^{n-1} \sigma^{i} f$ we may find a regular sequence $f_{1}, \ldots, f_{d}$ such that $f_{j}-\frac{1}{n} \sum_{i=0}^{n-1} \sigma^{i} f_{j}=f_{j}$ for $1 \leq j \leq m$ and $\sigma f_{j}=f_{j}$ for $m+1 \leq j \leq d$, for appropriate $m$. Fact (i) identifies $m$ elements which generate $I_{\sigma}$, and by (ii) we have $f_{j} \in I_{\sigma}$ for $1 \leq j \leq m$. Since $f_{1}, \ldots, f_{m}$ are linearly independent in $\mathfrak{m} / \mathfrak{m}^{2}$, the elements $f_{1}, \ldots, f_{m}$ generate $I_{\sigma}$.

Let $G$ be an algebraic group, and let $X$ be a stack. A $G$-action on $X$ is given by an action map $a: G \times X \rightarrow X$ together with an associativity 2-morphism $\gamma$ and identity 2-morphism $\beta$ satisfying compatibility conditions (associativity must satisfy a commutative cube, and identity must be compatible with associativity). Such an action yields a quotient stack $Y$ with map $Y \rightarrow B G$ such that $X$ is identified with $Y \times_{B G}$ Spec $k$. Suppose the action map is the projection map $p r_{2}: G \times X \rightarrow X$. Then the associativity 2-morphism is an automorphism $p r_{3}: G \times G \times X \rightarrow X$, and the action is trivial (i.e., $Y \simeq X \times B G$ ) if and only if $\gamma$ is obtained from $1_{p r_{3}}$ by composing with $\beta$. A general action is trivial if there is a 2-morphism $\delta: a \rightarrow p r_{2}$ such that if $\gamma^{\prime}$ and $\beta^{\prime}$ denote the compatibility morphisms obtained from $\gamma$ and $\beta$ respectively by 
applying $\delta$, then $\gamma^{\prime}$ is obtained from $1_{p r_{3}}$ by composing with $\beta^{\prime}$. So, for example, if $G$ is connected, then the $G$-action is trivial if and only if $a$ is 2 -isomorphic to $p r_{2}$. If we form the stabilizer diagram

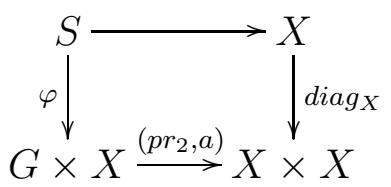

then $S$ has the structure of group object in the category of $X$-stacks, $\varphi$ is a group homomorphism, and if $G$ is connected then the action is trivial if and only if $\varphi$ admits a splitting.

For the remainder of this section, we assume the base field $k$ to be algebraically closed.

Suppose now $T$ is the one-dimensional torus $T=\mathbb{G}_{m}$, and suppose $T$ acts on $X=B H$, for some finite group $H$. We form the stabilizer diagram; the coarse moduli space to the component of the identity of the stabilizer $S$ is some $T^{\prime} \simeq \mathbb{G}_{m}$, with $T^{\prime} \rightarrow T$ unramified. If we consider the induced $T^{\prime}$-action on $X$, then $\varphi$ in (6.3.1) admits a splitting, and thus the action is trivial. Requiring a torus extension to trivialize the action on fixpoints accounts for the fractional weights that show up in calculations, e.g., in [Ko].

Proposition 6.3.4. Let $X$ be an integral Deligne-Mumford stack, and assume $X$ has finite stabilizer. Suppose the one-dimensional torus $T$ acts on $X$. Then there exists a $T$-stable closed substack $Z$ of $X$ and a positive integer $n$, prime to the characteristic of the base field, such that if $T^{\prime} \rightarrow T$ denotes the $n$-fold cover, then the induced action of $T^{\prime}$ on $Z$ is trivial, and the induced action of $T^{\prime}$ on $X \backslash Z$ has quasi-finite stabilizer. Moreover, if $X$ is smooth, then $Z$ is smooth.

Proof. Consider the stabilizer diagram (6.3.1), with $G=T$. Since $X$ has finite stabilizer, the fiber of $\varphi$ over a general point of $T$ maps finitely to $X$, and determines a closed substack $Z$ of $X$ (which we give the reduced substack structure). For suitable $n$, the $n$-fold covering torus $T^{\prime}$ acts trivially on the integral zero-dimensional substacks of $Z$. Consider now diagram (6.3.1) with $G=T^{\prime}$ and $X=Z$. The map from the connected component of the identity to $T^{\prime} \times Z$ is an isomorphism, and hence $T^{\prime}$ acts trivially on $Z$. The action of $T^{\prime}$ on $X \backslash Z$ clearly has quasi-finite stabilizer.

Now assume $X$ is smooth. We find $Z=\varphi\left(\varphi^{-1}(\{t\} \times X)\right)$ for all $t$ outside a finite subset of $T$, and in particular for $t$ equal to a primitive $r^{\text {th }}$ root of unity, for suitable $r$ prime to the characteristic of the base field. So, $Z$ is the fixed locus of a cyclic group action. The cyclic group action may be presented by the action of a generator $\sigma: X \rightarrow X$, together with a 2-morphism $\delta: \sigma^{r} \rightarrow 1_{X}$. Since the action on $Z$ is trivial, there exists a 2-morphism $\left.\sigma\right|_{Z} \rightarrow 1_{Z}$, compatible with $\delta$.

Let $f: U \rightarrow X$ be an étale atlas, and let $R=U \times_{X} U$. Replacing $U$ by the fiber product of $f, f \circ \sigma, \ldots$, we may assume that $\sigma$ is represented by an automorphism of 
$U$. The closed substack $Z$ of $X$ corresponds to a closed subscheme $Y$ of $U$. Suppose $u$ is a closed point of $Y$. Passing to the henselization $U^{h}$ of $U$ at $u$, we may assume $\left.\sigma\right|_{Y^{h}}=1_{Y^{h}}$. Thus we have $\sigma^{r}=1_{U^{h}}$. Hence the ideal $I$ of $\mathcal{O}_{U^{h}}$ corresponding to the closed subscheme $Y$ of $U$ satisfies $I \supset I_{\sigma}$, where $I_{\sigma}$ corresponds to the fixed locus of the action of $\sigma$ on $U^{h}$. Since $R$ contains an identity component, we also have $I \subset I_{\sigma}$. So, by Lemma $6.3 .3, \mathcal{O}_{u, Y}$ is regular, and thus $Z$ is smooth.

Theorem 6.3.5. Let $X$ be a Deligne-Mumford stack which has finite stabilizer, and suppose $T=\mathbb{G}_{m}$ acts on $X$. Let $A_{*}^{T} X$ denote $A_{*}$ of the stack quotient $[X / T]$. If $X^{T}$ denotes the fixed-point substack (which was called $Z$ in Proposition 6.3.4), and if we let $t=c_{1}(\mathcal{O}(1)) \in A_{*} B T$, then the inclusion $Z \rightarrow X$ induces an isomorphism

$$
A_{*} X^{T} \otimes_{\mathbb{Q}} \mathbb{Q}\left[t, t^{-1}\right] \rightarrow A_{*}^{T} X \otimes_{\mathbb{Q}[t]} \mathbb{Q}\left[t, t^{-1}\right] .
$$

Proof. Let $T^{\prime} \rightarrow T$ be the $n$-fold covering torus from Proposition 6.3.4. The action of $T^{\prime}$ on $U=X \backslash X^{T}$ has quasi-finite stabilizer, so the zeroth and first Chow homology groups of the stack quotient $A_{*}^{T} U$ and $\underline{A}_{*}^{T} U$ vanish in the dimension ranges indicated by Corollary 6.3.2.

It suffices to prove the theorem for the induced $T^{\prime}$-action on $X$, so we may assume $T$ acts trivially on $X^{T}$. Let $i: X^{T} \rightarrow X$ denote inclusion. Exactness of the localization sequence

$$
\underline{A}_{j}^{T} U \rightarrow A_{j} X^{T} \otimes_{\mathbb{Q}} \mathbb{Q}[t] \stackrel{i_{*}}{\rightarrow} A_{j}^{T} X \rightarrow A_{j}^{T} U \rightarrow 0
$$

implies that both the kernel and cokernel of $i_{*}$ are killed by suitable powers of $t$, and the theorem follows.

Let $X_{1}, \ldots, X_{m}$ denote the connected components of $X^{T}$, and let $j_{i}$ denote the corresponding inclusion maps, $j=1, \ldots, m$. Apparently, any $\alpha \in A_{*}^{T} X \otimes_{\mathbb{Q}[t]} \mathbb{Q}\left[t, t^{-1}\right]$ can be written uniquely as $\alpha=\sum_{j} i_{j *} \alpha_{j}$ with $\alpha_{j} \in A_{*} X_{j} \otimes \mathbb{Q}\left[t, t^{-1}\right]$. Now suppose $X$ is smooth. In analogy with the construction of [E-G1] we obtain a ring structure on $A_{*}^{T} X$. By Proposition 6.3.4, each $X_{i}$ is smooth, so $A_{*}^{T} X_{i} \simeq A_{*} X_{i} \otimes \mathbb{Q}\left[t, t^{-1}\right]$ also has a ring structure.

Writing $1=\sum_{j} i_{j *} \omega_{j}$ in $A_{*}^{T} X$, the identity $\alpha=\alpha \cdot 1$ implies the identities $\alpha_{j}=$ $\omega_{j} \cdot i_{j}^{*} \alpha$ in the ring $A_{*} X_{j} \otimes \mathbb{Q}\left[t, t^{-1}\right]$, for each $j$. In particular, $1=\omega_{j} \cdot c_{\text {top }}\left(N_{X_{j}} X\right)$, so we deduce

Corollary 6.3.6. Assume $X$ is a smooth Deligne-Mumford stack which has finite stabilizer, and suppose the one-dimensional torus $T$ acts on $X$ with fixed locus $X^{T}=$ $X_{1} \amalg \cdots \amalg X_{m}$. Then the equivariant normal bundle $c_{\mathrm{top}}\left(N_{X_{j}} X\right)$ is invertible in $A_{*} X_{j} \otimes \mathbb{Q}\left[t, t^{-1}\right]$ for each $j$, and we have

$$
\alpha=\sum_{j=1}^{m} i_{j *} \frac{i_{j}^{*} \alpha}{c_{\mathrm{top}}\left(N_{X_{j}} X\right)}
$$

for any $\alpha \in A_{*}^{T} X \otimes_{\mathbb{Q}[t]} \mathbb{Q}\left[t, t^{-1}\right]$. 


\section{References}

[A] M. Artin, Versal deformations and algebraic stacks, Invent. Math. 27 (1974), 165-189.

[B-F] K. Behrend and B. Fantechi, The intrinsic normal cone, Invent. Math. 128 (1997), 45-88.

[B11] S. Bloch, Algebraic cycles and higher K-theory, Adv. Math. 61 (1986), 267304.

[B12] S. Bloch, The moving lemma for higher Chow groups, J. Alg. Geom. 3 (1994), 537-568.

[D] P. Deligne, La formule de dualité globale, in M. Artin, A. Grothendieck, and J. L. Verdier, Théorie des Topos et Cohomologie Etale des Schémas $(S G A$ 4), t. 3, Lect. Notes in Math. 305, Springer-Verlag, Berlin, 1973, exposé XVIII.

[D-M] P. Deligne and D. Mumford, The irreducibility of the space of curves of given genus, Publ. Math. IHES 36 (1969), 75-109.

[E-G1] D. Edidin and W. Graham, Equivariant intersection theory, Invent. Math. 131 (1998), 595-634.

[E-G2] D. Edidin and W. Graham, Localization in equivariant intersection theory and the Bott residue formula, Am. J. Math. 120 (1998), 619-636.

[F] W. Fulton, Intersection Theory, Springer-Verlag, Berlin, 1984.

[Gi1] H. Gillet, Intersection theory on algebraic stacks and $Q$-varieties, J. Pure Appl. Algebra 34 (1984), 193-240.

[Gi2] H. Gillet, Riemann-Roch theorems for higher algebraic $K$-theory, Adv. Math. 40 (1981), 203-289.

[Gi3] H. Gillet, Some new Gysin homomorphisms for the Chow homology of varieties, Proc. London Math. Soc. (3) 50 (1985), 57-68.

[Gr1] A. Grothendieck, Descente fidèlement plate, in A. Grothendieck and M. Raynaud, Revêtements étales et groupe fondamental (SGA 1), Lect. Notes in Math 224, Springer-Verlag, Berlin, 1971, exposé VIII.

[Gr2] A. Grothendieck, Sur quelques propriétés fondamentales en théorie des intersections, in Anneaux de Chow et applications, Séminaire C. Chevalley $\mathbf{2}$ (1958), exposé 4. 
[EGA2] A. Grothendieck, Eléments de géométrie algébrique II: Etude globale élémentaire de quelques classes de morphisms. Publ. Math. IHES 8 (1961).

[EGA4] A. Grothendieck, Eléments de géométrie algébrique IV: Etude locale des schémas et des morphismes de schémas. Publ. Math. IHES 20 (1964), 24 (1965), 28 (1966), 32 (1967).

[Ki] S. Kimura, Fractional intersection and bivariant theory, Comm. Algebra 20 (1992), 285-302.

[Kn] D. Knutson, Algebraic Spaces, Lect. Notes in Math. 203, Springer-Verlag, Berlin, 1971.

[Ko] M. Kontsevich, Enumeration of rational curves via torus actions, in The moduli space of curves (Texel Island, 1994), pp. 335-368, Progr. Math. 129, Birkhäuser, Boston, 1995.

[Kr1] A. Kresch, Canonical rational equivalence of intersections of divisors, Invent. Math. (to appear).

[Kr2] A. Kresch, Chow homology for Artin stacks, Ph.D. thesis, Univ. of Chicago, 1998.

[L-MB] G. Laumon and L. Moret-Bailly, Champs Algébriques, Prépubl. math. de l'Université de Paris-Sud, 1995.

[M] D. Mumford, Towards an enumerative geometry of the moduli space of curves, in Arithmetic and Geometry, Vol. II, pp. 271-328, Progr. Math. 36, Birkhäuser, Boston, 1983.

[S] H. Sumihiro, Equivariant completion, II, J. Math. Kyoto Univ. 15 (1975), 573-605.

[V1] A. Vistoli, Alexander duality in intersection theory, Compositio Math. 70 (1989), 199-225.

[V2] A. Vistoli, Intersection theory on algebraic stacks and on their moduli spaces, Invent. Math. 97 (1989), 613-670.

Department of Mathematics

University of Pennsylvania

209 South 33rd Street

Philadelphia, PA 19104 